LBNL-54212

UCB-PTH-03/36

\title{
Holographic Theories of Electroweak Symmetry Breaking without a Higgs Boson
}

\author{
Gustavo Burdman ${ }^{a}$ and Yasunori Nomura ${ }^{a, b}$ \\ ${ }^{a}$ Theoretical Physics Group, Lawrence Berkeley National Laboratory, Berkeley, CA 94720 \\ ${ }^{b}$ Department of Physics, University of California, Berkeley, CA 94720
}

\begin{abstract}
Recently, realistic theories of electroweak symmetry breaking have been constructed in which the electroweak symmetry is broken by boundary conditions imposed at a boundary of higher dimensional spacetime. These theories have equivalent 4D dual descriptions, in which the electroweak symmetry is dynamically broken by non-trivial infrared dynamics of some gauge interaction, whose gauge coupling $\tilde{g}$ and size $N$ satisfy $\tilde{g}^{2} N \gtrsim 16 \pi^{2}$. Such theories allow one to calculate electroweak radiative corrections, including the oblique parameters $S, T$ and $U$, as long as $\tilde{g}^{2} N / 16 \pi^{2}$ and $N$ are sufficiently larger than unity. We study how the duality between the $4 \mathrm{D}$ and $5 \mathrm{D}$ theories manifests itself in the computation of various physical quantities. In particular, we calculate the electroweak oblique parameters in a warped 5D theory where the electroweak symmetry is broken by boundary conditions at the infrared brane. We show that the value of $S$ obtained in the minimal theory exceeds the experimental bound if the theory is in a weakly coupled regime. This requires either an extension of the minimal model or departure from weak coupling. A particularly interesting scenario is obtained if the gauge couplings in the 5D theory take the largest possible values - the value suggested by naive dimensional analysis. We argue that such a theory can provide a potentially consistent picture for dynamical electroweak symmetry breaking: corrections to the electroweak observables are sufficiently small while realistic fermion masses are obtained without conflicting with bounds from flavor violation. The theory contains only the standard model quarks, leptons and gauge bosons below $\simeq 2 \mathrm{TeV}$, except for a possible light scalar associated with the radius of the extra dimension. At $\simeq 2 \mathrm{TeV}$ increasingly broad string resonances appear. An analysis of top-quark phenomenology and flavor violation is also presented, which is applicable to both the weakly-coupled and strongly-coupled cases.
\end{abstract}




\section{Introduction}

One of the greatest mysteries in particle physics is the origin of electroweak symmetry breaking. In the standard model the electroweak symmetry is broken by a vacuum expectation value (VEV) of a Higgs field, which is driven by a non-trivial potential introduced to break the symmetry. However, once the theory is extrapolated to higher energies in a perturbative way, one finds that the Higgs mass squared parameter receives large radiative corrections of the order of the cutoff scale, destabilizing the electroweak scale. Therefore, it is quite natural to suspect that some non-trivial strong dynamics is responsible for electroweak symmetry breaking in a direct or indirect way. Such a consideration leads to theories where the electroweak symmetry is broken by a condensation caused by strong gauge dynamics [1] or theories where the Higgs boson arises as a composite state of some strong interaction [2]. In these theories the strength of the relevant gauge interaction is weaker at higher energies, and becomes non-perturbative only at lower energies by the renormalization group evolution. This triggers electroweak symmetry breaking at exponentially lower energies compared with the cutoff scale, thus evading the problem of the stability.

In this paper we explore alternative possibilities for "dynamical" theories of electroweak symmetry breaking. Suppose the gauge interaction, which is responsible for electroweak symmetry breaking and non-perturbative at the electroweak scale, stays very strong at higher energies stronger than that above which the conventional perturbation theory breaks down. Apparently, this does not provide any viable description of physics at energies higher than the electroweak scale. However, the presence of dualities between the 4D gauge theories and higher dimensional gravitational theories suggests that such a theory is described, in fact, by a higher dimensional theory where the electroweak symmetry is broken by the presence of a spacetime boundary. This relation becomes particularly concrete when the theory on the gravitational side is on an Anti de-Sitter (AdS) background [3], and it has been used to build models of a composite Higgs boson [4] and dynamical electroweak symmetry breaking [5, 6]. However, the background geometry of the gravitational theory may not necessarily be AdS, as in the models considered in $[7,8]$.

In this paper we consider theories of the kind described above, in which the holographic description of the theory relates a higher dimensional theory to some 4D "gauge theory". In particular, we study theories where the electroweak symmetry is broken "dynamically" without the presence of the physical Higgs boson - in the higher dimensional picture this corresponds to the theories where the electroweak symmetry is broken by boundary conditions imposed at a boundary of the spacetime. We mainly consider theories formulated in the AdS space, in which the electroweak symmetry is broken by boundary conditions imposed at the infrared (IR) 
brane $[5,6]$, but some of our analysis applies to more general theories such as the ones in flat space $[7,8]$. In the actual analysis we adopt the specific theory constructed in [6], which reproduces many successful features of the standard model including fermion mass generation and suppression of flavor changing neutral currents (FCNCs). This theory also allows us to control the scale of new physics, which corresponds in the 4D picture to the size (the number of "colors") of the gauge interaction, and thus represents a class of generic theories in 5D AdS space. We study electroweak radiative corrections and find that the constraints from precision electroweak measurements prefer gauge groups of smaller size, unless some additional contribution to the electroweak oblique parameters is introduced. This situation is similar to that in technicolor theories [9]. We elucidate how such a similarity arises in general theories with the electroweak symmetry broken by boundary conditions.

Although regarding electroweak corrections the situation in our theory is similar to that in technicolor, other aspects can be quite different. In particular, we expect that the theory does not have problems in general to obtain realistic fermion masses, correct vacuum alignment, and suppression of flavor violation. This implies that even the minimal theory may have a viable parameter region in which the size of the gauge group responsible for electroweak symmetry breaking is small, because the corrections to the electroweak observables become small there. We give an estimate for these corrections and find that they are in fact phenomenologically acceptable if the size of the gauge group is sufficiently small. Unfortunately, we find that this is the region where the theory looses its weakly coupled description, which prevents us to make a precise comparison with experiment. It also implies that we have to take into account stringy effects to construct a fully well-defined and ultraviolet (UV) completed theory. However, given the presence of an effective field theoretic model and a freedom of taking a certain limit, it does not seem so implausible to expect that this type of theories does in fact exist. The experimental signatures of such theories are quite distinct. There is essentially no new state appearing below a few $\mathrm{TeV}(\approx 2-3 \mathrm{TeV})$ other than the standard model gauge bosons and quarks and leptons; in particular, there is no Higgs boson. We then see new states, most of which are associated with string states, at the scale of a few $\mathrm{TeV}$. These states arise, in the $4 \mathrm{D}$ picture, from the non-trivial dynamics of the new strong gauge interaction. The unitarity of the theory is cured by these states and the tail of that physics, which may also be seen in scattering experiments at somewhat lower energies than their actual masses.

The organization of the paper is as follows. In the next section we give a general discussion on theories where the electroweak symmetry is broken "dynamically". We argue that conventional technicolor-type theories and extra dimensional theories with boundary condition electroweak symmetry breaking are related in a certain way in the space of the gauge coupling, and we elucidate how the electroweak corrections in these theories have some similarities. In section 3 we 
present the model we study, constructed in the truncated 5D AdS space. Electroweak corrections are studied in section 4, where we calculate the electroweak oblique parameters and compare with experiment. We discuss two possible scenarios which can be phenomenologically viable. In section 5 we study the top quark sector and its related phenomenology. Flavor violation is also studied there. Conclusions and discussion are given in section 6 .

\section{Holography and Electroweak Symmetry Breaking}

In this paper we mostly study theories formulated in the truncated 5D AdS space. This type of theories can provide an understanding of a large hierarchy between the Planck and the electroweak scales through the AdS warp factor [10]. Before presenting an explicit model and going into the detailed calculation, however, we here start by some general discussion on theories of "dynamical" electroweak symmetry breaking. These include conventional technicolor [1, 11] and walking technicolor [12] theories, as well as theories based on extra dimensions such as the ones on flat $[7,8]$ or warped $[5,6]$ geometries. We will see that these theories are related in a certain way in the space of the gauge coupling and the size of the gauge group.

In order to break the electroweak symmetry dynamically in the IR, we need some gauge interaction $G$ that becomes non-perturbative at low energies. We denote the coupling and the size (the number of "colors") of this gauge interaction as $\tilde{g}$ and $N$, respectively. In general $4 \mathrm{D}$ theories, the coupling $\tilde{g}$ runs with energy. Suppose now that $G$ is a usual asymptoticallyfree gauge interaction. In this case the theory is weakly coupled at the UV: the parameter $\kappa \equiv \tilde{g}^{2} N / 16 \pi^{2}$, which is the loop expansion parameter of the gauge theory, satisfies $\kappa \ll 1$. In the IR the parameter $\kappa$ evolves to larger values, and at some scale becomes $\kappa \simeq 1$, where the theory exhibits non-trivial dynamical phenomena such as chiral symmetry breaking. Then, if some fields of the $G$ sector are charged under $S U(2)_{L} \times U(1)_{Y}$, the electroweak symmetry can be broken at this scale. This is the situation in conventional technicolor theories $[1,11]$. Alternatively, $\kappa$ could approach to some constant value close to but somewhat smaller than 1 at the UV, instead of $\kappa \rightarrow 0$ (or decrease only very slowly near $\Lambda$ ). Such is the case in walking technicolor theories [12].

Now, let us consider very different possibilities. At the electroweak scale the interaction $G$ induces non-trivial dynamical phenomena. Is it then possible for $\kappa$ to take larger values than 1 at the UV, instead of smaller values? At first sight, this does not make sense, because the loop expansion parameter of the theory is larger than unity at the UV - in fact, the description based on the 4D gauge theory can completely break down. However, in the parameter region $\kappa \gtrsim 1$, another (sometimes weakly coupled) description of the theory could emerge. Suppose we take the limit $N \gg 1$, keeping $\kappa$ fixed to some value larger than unity. In this case, the 
loop diagrams are sorted by the topology of the graphs and we find that diagrams with different topologies correspond to ones having different powers of $N$, allowing us to expand the theory in powers of $1 / N$ [13]. Since this expansion is reminiscent to the loop expansion by the topology of the world-sheet in string theory, the gravitational description of the theory emerges. This dual gravitational theory possesses spacetime dimensions larger than four, as required by string theory. The parameter $1 / N$ plays a role of the (string) coupling constant, while the value of $\kappa$ turns out to be a measure of the importance of string corrections [3]. Therefore, for sufficiently large values of $N$ the gravitational description is weakly coupled. For $\kappa \gg 1$ the string corrections are small, corresponding to the region where the curvature scale of the gravitational background is much smaller than the string scale, while for $\kappa \simeq 1$ the string effects are important.

We can now consider the following scenario. At the UV the theory has $\kappa$ which is close to but somewhat larger than 1 . The coupling $\kappa$ is almost constant (conformal) over a wide energy interval, but at some IR scale this conformality breaks down, triggering non-trivial gauge dynamics. In particular, it induces chiral symmetry breaking and consequently breaks the electroweak symmetry. In the dual gravitational description, this theory will look like a 5D theory on AdS, as suggested by the isomorphism between the 4D conformal group and the isometry of 5D AdS space [3]. The scale of AdS curvature is smaller than the string scale if $\kappa$ is larger than unity. The non-trivial IR dynamics is then represented by the presence of a boundary in the spacetime, beyond that point the gravitational description disappears, i.e. the gauge theory "confines". We can thus conjecture that the theory has a 5D description, compactified on a warped $S^{1} / Z_{2}$ orbifold with the boundary condition on the IR brane breaking the electroweak symmetry. ${ }^{1}$ This type of theories have been considered in $[5,6]$ and will be described in the next section. The simple relation between the 4D and 5D theories holds only at energies lower than the AdS curvature scale $k$, beyond which the models of $[5,6]$, for instance, appear intrinsically five dimensional. This scale, however, is much higher than the electroweak scale (close to the Planck scale). An interesting point is that these theories allow a large energy interval above the electroweak scale, in which the gravitational description does not break down. This is due to the large warp factor of AdS, or the near conformal nature of the theory.

Models on 5D flat space, such as the ones considered in $[7,8]$, can be obtained from models on AdS by taking the limit that the AdS curvature scale is small, $k \rightarrow 0$. In this case, however, the scale where the simple 4D/5D correspondence breaks down becomes close to the electroweak scale. In fact, there is no energy interval where the field theoretic correspondence works, and the theory appears five dimensional right above the scale of dynamical electroweak symmetry

\footnotetext{
${ }^{1}$ Strictly speaking, this will be the case only for certain special $4 \mathrm{D}$ gauge theories. For instance, weaklycoupled 5D theories have a feature that the resonances having spin larger than two are much heavier than the others, which is not a property of generic large $N$ gauge theories.
} 


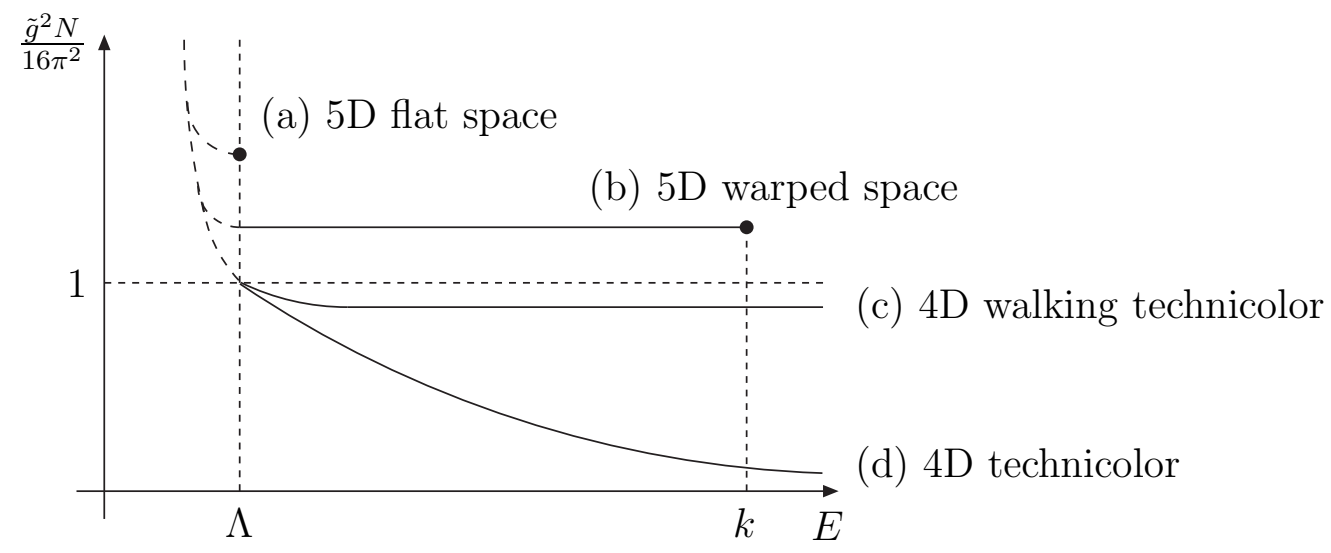

Figure 1: Schematic description for the evolution of the coupling parameter $\kappa \equiv \tilde{g}^{2} N / 16 \pi^{2}$ in various theories of dynamical electroweak symmetry breaking. The behaviors of (a), (b), (c), and (d) represent those of 5D flat space, 5D warped space, walking technicolor and technicolor theories, respectively.

breaking. Some 4D interpretation of the theory, however, may be possible for some purposes that do not involve physics much above the electroweak scale.

The schematic behavior of each of these four types of theories is depicted in Fig. 1 as a function of the energy $E$. The theories (a), (b), (c), and (d) correspond, respectively, to 5D flat space, 5D warped space, walking technicolor, and technicolor theories. The parameter $\Lambda$ represents the scale where $G$ exhibits non-trivial IR dynamics, especially chiral symmetry breaking, which is roughly the mass of the first resonance state and not much different from the electroweak scale. For $\kappa \gg 1$, the predictions of the theory depend quite little on the value of $\kappa$, because the theory admits an expansion in $1 / \kappa$, with higher order terms corresponding to stringy corrections, so that physical quantities are almost determined by the first term in the expansion, which is independent of $\kappa$. This implies that electroweak oblique corrections, whose contributions come from $E \approx \Lambda$, can have similar structure in the four types of theories, all of which have $\kappa \gtrsim 1$ at the scale $\Lambda$. In particular, the size of the corrections essentially depends only on a single parameter $N$. However, there is an important difference between the theories of the types (a),(b) and (c),(d). In theories (a) and (b) the electroweak oblique parameters are calculable for $N \gg 1$, as the higher order terms in the double expansions in $1 / N$ and $1 / \kappa$ are both negligible, while theories (c) and (d) do not have such calculational powers because $\kappa \simeq 1$ at $E \simeq \Lambda$. This calculability, however, is lost in theories (a) and (b) when we make $N$ or $\kappa$ smaller. For the physics occurring above $\Lambda$, such as fermion mass generation, the physical pictures could be quite different in different types of theories. 
In the following sections we focus on theories in the truncated 5D AdS space (the type (b) theories in Fig. 1). In particular, we consider electroweak radiative corrections in these theories in section 4. As explained, their structure is expected to be similar to the one in technicolor. Our analysis based on the large $N$ expansion will explicitly demonstrate that they are, in fact, very similar. $^{2}$ While we perform the analysis for the case of warped space theories, our qualitative results given in section 4 also apply to the case of flat space theories by replacing the AdS curvature $k$ by the size of the flat extra dimension: $k \rightarrow 1 / \pi R$.

\section{The Model}

In this section we review the model constructed in Ref. [6], which we will explicitly work on in the rest of the paper. This model reproduces many successful features of the standard model, including fermion mass generation and suppression of FCNCs. The gauge sector of the model is quite generic and contains, for example, that of [5] as a special point in the parameter space.

The theory is formulated in the 5D warped space with the extra dimension compactified on $S^{1} / Z_{2}$. The metric is given by

$$
d s^{2} \equiv G_{M N} d x^{M} d x^{N}=e^{-2 k|y|} \eta_{\mu \nu} d x^{\mu} d x^{\nu}+d y^{2}
$$

where $y$ is the coordinate of the fifth dimension and the physical space is taken to be $0 \leq y \leq \pi R$. We take the AdS curvature scale $k$ to be around the 4D Planck scale, and we choose the radius $R$ to be $k R \sim 10$. The scale of the IR brane $k^{\prime}$ (denoted by $T$ in [6]) is then given by $k^{\prime} \equiv k e^{-\pi k R} \sim \mathrm{TeV}[10]$. The fundamental (cutoff) scale of the theory is denoted by $M_{*}$, which is taken to be $M_{*} \gtrsim k$, and the IR cutoff scale is defined by $M_{*}^{\prime} \equiv M_{*} e^{-\pi k R}$.

The bulk gauge group is $S U(3)_{C} \times S U(2)_{L} \times S U(2)_{R} \times U(1)_{X}$. It is broken by boundary conditions imposed at the $y=0$ brane (the Planck brane):

$$
\partial_{y} A_{\mu}^{L a}=0, \quad A_{\mu}^{R 1,2}=0, \quad \partial_{y}\left(\frac{1}{g_{R}^{2}} A_{\mu}^{R 3}+\frac{1}{g_{X}^{2}} A_{\mu}^{X}\right)=0, \quad A_{\mu}^{R 3}-A_{\mu}^{X}=0,
$$

and at the $y=\pi R$ brane (the TeV brane):

$$
\partial_{y}\left(\frac{1}{g_{L}^{2}} A_{\mu}^{L a}+\frac{1}{g_{R}^{2}} A_{\mu}^{R a}\right)=0, \quad A_{\mu}^{L a}-A_{\mu}^{R a}=0, \quad \partial_{y} A_{\mu}^{X}=0,
$$

with the $A_{5}$ 's obeying Dirichlet (Neumann) boundary conditions if the corresponding $A_{\mu}$ 's obey Neumann (Dirichlet) boundary conditions [5, 7] (these boundary conditions are slightly modified

\footnotetext{
${ }^{2}$ A numerical coincidence between technicolor and 5D flat theories found in [8] also suggests that this similarity could be a very precise one.
} 
when brane-localized gauge kinetic terms are introduced at $y=0$ and $y=\pi R$ ). At the Planck brane, the bulk gauge group is broken to the standard-model gauge group $S U(3)_{C} \times S U(2)_{L} \times$ $U(1)_{Y}$, where $U(1)_{Y}$ is a linear combination of $U(1)_{X}$ and the $T_{3}$ direction of $S U(2)_{R}$, while at the TeV brane, $S U(2)_{L} \times S U(2)_{R}$ is broken to the $S U(2)$ diagonal subgroup. Combining the breaking at the both branes, the unbroken gauge group at low energies becomes $S U(3)_{C} \times U(1)_{\mathrm{EM}}$, where $U(1)_{\mathrm{EM}}$ refers to electromagnetism. In particular, the electroweak symmetry is broken by the boundary conditions at the TeV brane.

We can view the above boundary conditions as the limiting case of the following brane Higgs breaking. We introduce a scalar field $\Sigma(\mathbf{1}, \mathbf{1}, \mathbf{2}, 1 / 2)$ on the Planck brane and $H\left(\mathbf{1}, \mathbf{2}, \mathbf{2}^{*}, 0\right)$ on the $\mathrm{TeV}$ brane, where the numbers in the parentheses represent gauge quantum numbers under $S U(3)_{C} \times S U(2)_{L} \times S U(2)_{R} \times U(1)_{X}$. Now, suppose that these fields have VEVs:

$$
\langle\Sigma\rangle=\left(\begin{array}{c}
0 \\
v_{\Sigma}
\end{array}\right), \quad\langle H\rangle=\left(\begin{array}{cc}
v_{H} & 0 \\
0 & v_{H}
\end{array}\right) .
$$

We then find that for $v_{\Sigma}, v_{H} \rightarrow \infty$, the phenomenology of this Higgs-breaking theory becomes identical to that of the boundary-condition breaking theory [14]. In particular, the physical Higgs bosons arising from $\Sigma$ and $H$ decouple for large $v_{\Sigma}$ and $v_{H}$, so that there is no scalar particle remaining in the spectrum in this limit.

The kinetic terms for the gauge fields are given by

$$
\begin{aligned}
& \mathcal{S}=\int d^{4} x \int d y\left[\sqrt { - G } \left\{-\frac{1}{4 g_{L}^{2}} g^{M P} g^{N Q} \sum_{a=1}^{3} F_{M N}^{L a} F_{P Q}^{L a}-\frac{1}{4 g_{R}^{2}} g^{M P} g^{N Q} \sum_{a=1}^{3} F_{M N}^{R a} F_{P Q}^{R a}\right.\right. \\
& \left.\left.-\frac{1}{4 g_{X}^{2}} g^{M P} g^{N Q} F_{M N}^{X} F_{P Q}^{X}\right\}+\delta(y)\left\{-\frac{1}{4 \tilde{g}_{L}^{2}} \sum_{a=1}^{3} F_{\mu \nu}^{L a} F_{\mu \nu}^{L a}-\frac{1}{16 \tilde{g}_{Y}^{2}}\left(F_{\mu \nu}^{R 3}+F_{\mu \nu}^{X}\right)\left(F_{\mu \nu}^{R 3}+F_{\mu \nu}^{X}\right)\right\}\right],(5)
\end{aligned}
$$

where $F_{M N}^{L a}, F_{M N}^{R a}$ and $F_{M N}^{X}$ are the field-strength tensors for $S U(2)_{L}, S U(2)_{R}$ and $U(1)_{X}$, and $g_{L}$, $g_{R}$ and $g_{X}$ are the 5D gauge couplings having mass dimensions $-1 / 2 ; a$ is the indices for the adjoint representation of $S U(2)$. Here, we have included Planck-brane localized gauge kinetic terms, which are radiatively generated and generically have coefficients of order $\left(b / 8 \pi^{2}\right) \ln \left(k / k^{\prime}\right) \sim 1$. TeV-brane localized gauge kinetic terms are considered in the next section. We have omitted the gauge kinetic terms for $S U(3)_{C}$ in the above expression, since they are irrelevant for our discussion below.

The quarks and leptons are introduced in the bulk with the representations:

$$
\begin{array}{lc}
q(\mathbf{3}, \mathbf{2}, \mathbf{1}, 1 / 6), & \bar{u}=\left.\psi_{\bar{u}}\left(\mathbf{3}^{*}, \mathbf{1}, \mathbf{2},-1 / 6\right)\right|_{T_{3}^{R}=-1 / 2}, \quad \bar{d}=\left.\psi_{\bar{d}}\left(\mathbf{3}^{*}, \mathbf{1}, \mathbf{2},-1 / 6\right)\right|_{T_{3}^{R}=1 / 2}, \\
l(\mathbf{1}, \mathbf{2}, \mathbf{1},-1 / 2), & \bar{e}=\left.\psi_{\bar{e}}(\mathbf{1}, \mathbf{1}, \mathbf{2}, 1 / 2)\right|_{T_{3}^{R}=1 / 2}, \quad\left[\bar{n}=\left.\psi_{\bar{n}}(\mathbf{1}, \mathbf{1}, \mathbf{2}, 1 / 2)\right|_{T_{3}^{R}=-1 / 2}\right]
\end{array}
$$

where $q, \bar{u}, \bar{d}, l, \bar{e}$ and $\bar{n}$ are Dirac fermions and the numbers in the parentheses represent gauge quantum numbers under $S U(3)_{C} \times S U(2)_{L} \times S U(2)_{R} \times U(1)_{X} ; T_{3}^{R}= \pm 1 / 2$ represents the $T_{3}=$ 
$\pm 1 / 2$ component of the $S U(2)_{R}$ doublet. With the extra dimension compactified on $S^{1} / Z_{2}$, we can arrange the boundary conditions such that only the left-handed components of $q, \psi_{\bar{u}}, \psi_{\bar{d}}, l$ and $\psi_{\bar{e}}$ possess zero modes (also $\psi_{\bar{n}}$ if we introduce them to induce small neutrino masses through the see-saw mechanism; see $[6,15])$. Moreover, introducing the Planck-brane localized left-handed fermions $\psi_{\bar{u}}^{\prime}(\mathbf{3}, \mathbf{1}, \mathbf{1},-1 / 3), \psi_{\bar{d}}^{\prime}(\mathbf{3}, \mathbf{1}, \mathbf{1}, 2 / 3)$ and $\psi_{\bar{e}}^{\prime}(\mathbf{1}, \mathbf{1}, \mathbf{1}, 0)$, and the couplings $\delta(y)\left[\psi_{\bar{u}} \psi_{\bar{u}}^{\prime} \Sigma+\right.$ $\psi_{\bar{d}} \psi_{\bar{d}}^{\prime} \Sigma^{\dagger}+\psi_{\bar{e}} \psi_{\bar{e}}^{\prime} \Sigma^{\dagger}$, we can make the unwanted zero modes from the $T_{3}^{R}=1 / 2$ component of $\psi_{\bar{u}}$ and the $T_{3}^{R}=-1 / 2$ components of $\psi_{\bar{d}}$ and $\psi_{\bar{e}}$ heavy to get masses of order $k{ }^{3}$ The low-energy matter content is then precisely that of the standard-model quarks and leptons, which arise as the zero modes of $q, \bar{u}, \bar{d}, l$ and $\bar{e}$. The quark and lepton masses arise from the couplings introduced on the $\mathrm{TeV}$ brane

$$
\mathcal{S}=\int d^{4} x \int d y \delta(y-\pi R) \sqrt{-g_{\text {ind }}}\left[y_{u} q \psi_{\bar{u}} H+y_{d} q \psi_{\bar{d}} H+y_{e} l \psi_{\bar{e}} H+\text { h.c. }\right],
$$

where we have suppressed the generation index. As the up-type quark, down-type quark, and charged lepton masses arise from three independent couplings, $y_{u}, y_{d}$ and $y_{e}$, there are no unwanted relations among them coming from $S U(2)_{R}$.

The wavefunction profiles for the zero modes of the quark and lepton fields are controlled by the $5 \mathrm{D}$ bulk mass parameters for these fields, which we parameterize as $\mathcal{L}_{5 \mathrm{D}} \supset-c k \bar{\Psi} \Psi$ where $\Psi$ represents generic 5D (Dirac) fermions. For $c>1 / 2(c<1 / 2)$ the wavefunction for the lefthanded zero-mode fermion is localized to the Planck $(\mathrm{TeV})$ brane. We take parameters $c$ to be larger than $1 / 2$, at least for the first-two generation fermions. This makes the non-universality of the $W$ - and $Z$-boson couplings to these fields very small so that the theory is phenomenologically viable [6], and could also provide a partial understanding of the flavor structure of the fermion mass matrices and suppression of the flavor violation arising from the TeV-brane operators [17]. The $c$ parameters for the third generation fermions are the theme of section 5 , where the nonuniversality of the fermion gauge couplings is also discussed further.

We can now calculate the masses and couplings of the electroweak gauge bosons, $W, Z$ and $\gamma$. Assuming $1 / g_{L}^{2} \sim 1 / g_{R}^{2} \sim 1 / g_{X}^{2} \sim 1 / \pi R$ and $\tilde{g}_{L} \sim \tilde{g}_{Y} \sim 1$, we find that these masses and couplings take exactly the same form as that of the standard model, at the leading order in $1 / \pi k R$ and in $k^{\prime} / k$. Denoting the standard-model $S U(2)_{L}$ and $U(1)_{Y}$ couplings as $g$ and $g^{\prime}$ and the Higgs-field VEV as $v \simeq 175 \mathrm{GeV}$, the correspondence between the two theories are given by $[6]$

$$
\frac{1}{g^{2}}=\frac{\pi R}{g_{L}^{2}}+\frac{1}{\tilde{g}_{L}^{2}}, \quad \frac{1}{g^{\prime 2}}=\frac{\pi R}{g_{R}^{2}}+\frac{\pi R}{g_{X}^{2}}+\frac{1}{\tilde{g}_{Y}^{2}}, \quad v^{2}=\frac{4 k^{\prime 2}}{\left(g_{L}^{2}+g_{R}^{2}\right) k} .
$$

\footnotetext{
${ }^{3}$ This also makes the theory anomaly free together with the introduction of appropriate Chern-Simons terms [16].
} 
Thus, for given values of the brane couplings, $\tilde{g}_{L}$ and $\tilde{g}_{Y}$, we have two relations on the three bulk gauge couplings, $g_{L}, g_{R}$ and $g_{X}$, so that we can calculate various quantities in terms of a single free parameter, which we take to be $g_{R}^{2} / g_{L}^{2}$ (an introduction of TeV-brane gauge kinetic terms will give a few extra parameters). For instance, the value of $k^{\prime}$ is determined by the last equation of Eqs. (8), which in turn gives the masses of the Kaluza-Klein (KK) gauge bosons

$$
m_{n} \simeq \frac{\pi}{2}\left(n+\frac{1}{2}\right) k^{\prime}
$$

where $n=1,2,3,4 \cdots$ for the $W$ and $Z$ towers and $n=1,3,5, \cdots$ for the $\gamma$ and gluon towers [6].

What are values of the Planck-brane gauge couplings? In Eqs. (8) we have to use values of $\tilde{g}_{L}$ and $\tilde{g}_{Y}$ appropriately normalized at the scale around TeV. Since the running of the Planck-brane gauge couplings is determined by the zero modes (elementary fields in the dual picture) of the theory [18], we can write $1 / \tilde{g}_{L}^{2}$ and $1 / \tilde{g}_{Y}^{2}$ as

$$
\frac{1}{\tilde{g}_{L}^{2}}=\frac{1}{\tilde{g}_{L, 0}^{2}}+\frac{b_{L}}{8 \pi^{2}} \ln \left(\frac{k}{k^{\prime}}\right), \quad \frac{1}{\tilde{g}_{Y}^{2}}=\frac{1}{\tilde{g}_{Y, 0}^{2}}+\frac{b_{Y}}{8 \pi^{2}} \ln \left(\frac{k}{k^{\prime}}\right),
$$

where $\left(b_{L}, b_{Y}\right) \simeq(-10 / 3,20 / 3)$ in the present theory. The couplings $\tilde{g}_{L, 0}$ and $\tilde{g}_{Y, 0}$ represent the running couplings of the elementary $S U(2)_{L}$ and $U(1)_{Y}$ gauge bosons at the scale $k$. In general, these couplings are free parameters of the theory and cannot be calculated in the effective theory.

One natural possibility is to assume that the elementary sector of the theory is strongly coupled at the scale $k$, in which case the bare parameters, $\tilde{g}_{L, 0}$ and $\tilde{g}_{Y, 0}$, are estimated to be $1 / \tilde{g}_{L, 0}^{2} \sim 1 / \tilde{g}_{Y, 0}^{2} \sim 1 / 16 \pi^{2}$ through naive dimensional analysis (NDA) [19]. This is the case considered in [6] (and in [5]), leading to the situation that the free parameters in the gauge sector of the theory are effectively only $g_{R}^{2} / g_{L}^{2}$ and $M_{*} / k$ (and TeV-brane localized kinetic terms). Note, however, that, contrary to the flat space case, in warped space theories the strong-coupling requirement for the Planck-brane operators is independent from the strong-coupling requirement for the bulk and TeV-brane operators. For instance, it is completely natural to assume that all the bulk and TeV-brane operators scale according to NDA while couplings at the Planck brane are weak. (In the dual 4D picture, this is equivalent to requiring that the $G$ sector does not contain any small or large dimensionless parameter other than the size of the gauge group, while the elementary sector is weakly coupled.) In this case the observed $4 \mathrm{D}$ gauge couplings are almost entirely given by the Planck-brane couplings, $1 / g^{2} \simeq 1 / \tilde{g}_{L}^{2}$ and $1 / g^{\prime 2} \simeq 1 / \tilde{g}_{Y}^{2}$, and the bulk gauge couplings take the values determined by NDA, $1 / g_{L}^{2} \sim 1 / g_{R}^{2} \sim 1 / g_{X}^{2} \sim M_{*} / 16 \pi^{3}$ (a similar scenario has been considered in flat space in [8]). ${ }^{4}$ In either case the largest value

\footnotetext{
${ }^{4}$ Here we do not bother the difference between the 4D loop factor $16 \pi^{2}$ and the $5 \mathrm{D}$ loop factor $24 \pi^{3}$ too much, and adopt a somewhat "conservative" estimate using $16 \pi^{3}$. This gives a strong coupling value for the $4 \mathrm{D}$ gauge coupling, $g_{4 \mathrm{D}} \simeq 4 \pi$, when the IR cutoff $M_{*}^{\prime}$ is lowered to the mass of the first KK resonance, $M_{*}^{\prime} \simeq \pi k^{\prime}$ (see the last paper in [19]). We also do not include group theoretical factors for NDA because the bulk gauge groups (i.e. $S U(2)_{L}, S U(2)_{R}$ and $U(1)_{X}$, not $\left.G\right)$ are small.
} 
of $k^{\prime}$ is determined by the parameter $M_{*} /(\pi k)$ as $\left.k^{\prime}\right|_{\max } \approx(1 \sim 1.5 \mathrm{TeV})\left(\pi k / M_{*}\right)^{1 / 2}$, which is translated into the maximum value for the lowest $\mathrm{KK}$ gauge boson mass $\left.m_{1}\right|_{\max } \approx(2.5 \sim$ $3.5 \mathrm{TeV})\left(\pi k / M_{*}\right)^{1 / 2}$. If we require that the theory admits a weakly coupled description, e.g. $M_{*} /(\pi k) \gtrsim 3$, this gives $\left.k^{\prime}\right|_{\max } \approx(600 \sim 900) \mathrm{GeV}$ and $\left.m_{1}\right|_{\max } \approx(1.5 \sim 2) \mathrm{TeV}$.

In the next section we consider corrections to the electroweak observables in the present theory. Our analysis applies regardless of the values of $\tilde{g}_{L, 0}$ and $\tilde{g}_{Y, 0}$, and thus to either of the two cases described above.

\section{Electroweak Corrections from the Gauge Sector}

In this section we discuss the structure of the corrections to electroweak observables in the theory presented in the previous section. We concentrate here on the corrections from the pure gauge sector and leave those from the matter sector to the next section. We consider the electroweak oblique parameters $S, T$ and $U[9]$ and give estimates for them. We elucidate how the $4 \mathrm{D}$ dual picture provides a qualitative understanding of the structure of these corrections. We then calculate the leading corrections in the 5D picture and compare them with the results of 4D considerations, which quantitatively demonstrates the duality between the two theories. We find that, if we stick to the presence of a weakly coupled gravitational description of the theory, the model gives somewhat larger (positive) values of $S$ than those allowed by precision electroweak measurements. There are essentially two ways out of this unpleasant situation. One is to extend the model such that it has a sector giving a negative value of $S$ canceling the positive contribution. The other is to give up the weakly coupled description of the theory. In particular, we argue that once we depart from the weakly coupled description, the theory could avoid constraints from the precision measurements.

Some of the discussions in this section overlap with those in [8], which explicitly considers these issues in a flat space model with some discussions on general gravitational backgrounds. Our explicit result for the warped space model agrees with the expectation given in [8].

\subsection{Structure of electroweak corrections}

We start with the 4D dual picture of the theory. As discussed in section 2, we can relate the theory described in the previous section to a purely $4 \mathrm{D}$ theory through the AdS/CFT correspondence $[3,20]$. In this $4 \mathrm{D}$ dual picture, the theory below $k \sim M_{\mathrm{pl}}$ contains a gauge interaction with the group $G$, whose coupling evolves very slowly over a wide energy interval below $k$. This $G$ gauge sector possesses a global $S U(3)_{C} \times S U(2)_{L} \times S U(2)_{R} \times U(1)_{X}$ symmetry whose $S U(3)_{C} \times S U(2)_{L} \times U(1)_{Y}$ subgroup is gauged, where $U(1)_{Y}$ is a linear combination of 


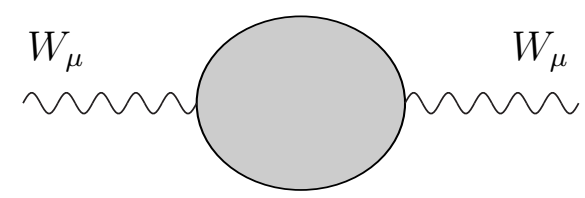

Figure 2: The diagram contributing to the $W$ boson propagators. Similar diagrams exist with one or two external $W_{\mu}$ 's replaced by $B_{\mu}$.

$U(1)_{X}$ and the $T_{3}$ direction of $S U(2)_{R}$. Therefore, the theory in this energy interval appears as $S U(3)_{C} \times S U(2)_{L} \times U(1)_{Y} \times G$ gauge theory with the quarks and leptons transforming under $S U(3)_{C} \times S U(2)_{L} \times U(1)_{Y}$. At the $\mathrm{TeV}$ scale the gauge interaction of $G$ exhibits non-trivial IR phenomena, producing resonances of masses of order $\mathrm{TeV}$. These resonances have a tower structure. In particular, there are towers of spin-1 fields which have the quantum numbers of $W$, $Z$ and $\gamma$. These towers then mix with the elementary gauge bosons of the weakly gauged $S U(2)_{L}$ and $U(1)_{Y}$ groups. The resulting spectrum consists of towers of gauge bosons with the quantum numbers of $W$ and $Z$, whose lowest states are massive and identified as the standard-model $W$ and $Z$ bosons, and a tower of $U(1)$ gauge bosons, whose lowest mode is massless and identified with the photon. These towers of mass eigenstates are dual to the $W, Z$ and $\gamma$ KK towers in the $5 \mathrm{D}$ picture. The electroweak gauge group $S U(2)_{L} \times U(1)_{Y}$ is dynamically broken and the masses of the $W$ and $Z$ bosons and the quarks and leptons are generated.

How do the corrections to electroweak gauge boson propagators arise in the 4D picture? We concentrate here on the effect from spin-1 resonances and leave the consideration of the other effects to the next section. At leading order, the corrections arise from the diagrams such as the one given in Fig. 2, where the gray disk at the center of the diagram represents contributions from the strongly interacting $G$ sector. We have drawn only the diagram giving corrections to the $S U(2)_{L}$ gauge boson, $W_{\mu}^{a}$, but similar diagrams also exist with one or two external $W_{\mu}$ 's replaced by the $U(1)_{Y}$ gauge boson, $B_{\mu}$. These diagrams give a contribution to the $S$ parameter (specifically, it arises from the diagram having $W_{\mu}$ for one external line and $B_{\mu}$ for the other). To evaluate the contribution, we must know what this gray disk actually means. For a sufficiently large value of $N$, this leading order contribution comes from the sum of a series of diagrams given in Fig. 3 (the planar diagrams [13]). In the figure we have given the size of contributions from each diagram. Writing the contribution from all the diagrams in the form $\left(N / 16 \pi^{2}\right) f\left(\tilde{g}^{2} N / 16 \pi^{2}\right)$ where $f(x)$ is some function, we expect that the gray disk gives a contribution of order $N / 16 \pi^{2}$ in Fig. 2 and thus changes the coefficients of the gauge kinetic terms from $1 / g^{2}$ to $1 / g^{2}+c N / 16 \pi^{2}$, 


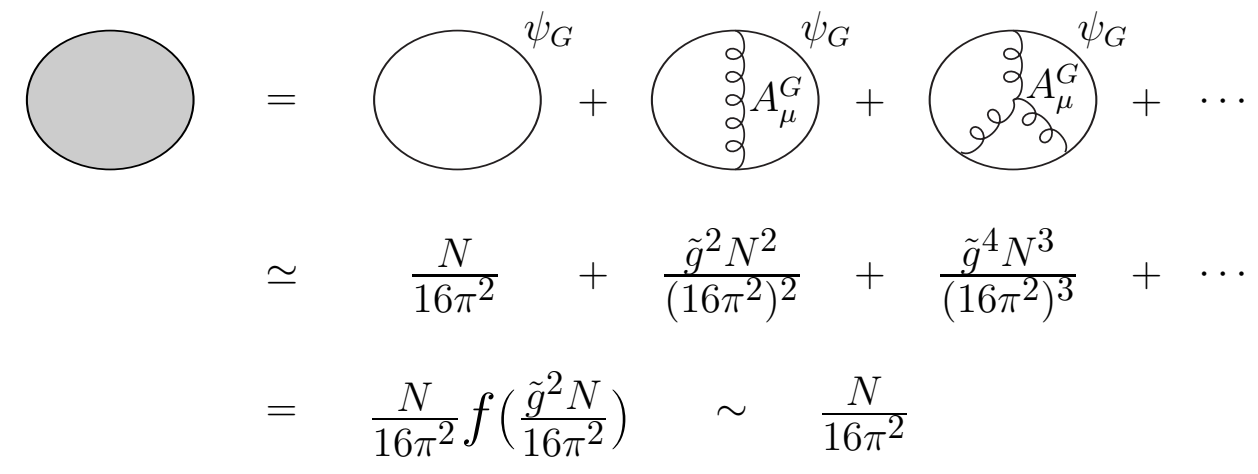

Figure 3: The diagrams represented by the gray disk. Here, $\psi_{G}$ and $A_{\mu}^{G}$ represent matter and gauge fields of the strongly-coupled $G$ sector. The size of each diagram is also shown. The contribution from this set of diagrams will be of order $N / 16 \pi^{2}$.

where $g$ represents generic electroweak gauge couplings and $c$ is a numerical factor of $O(1){ }^{5}$ This gives a contribution to the $S$ parameter

$$
S \simeq \frac{N}{\pi}
$$

since $S$ is given by $S \equiv 16 \pi\left(\Pi_{33}^{\prime}-\Pi_{3 Q}^{\prime}\right)$, where $\Pi_{X Y}^{\prime}$ are the corrections to the gauge kinetic terms defined by $\mathcal{L}=-(1 / 4)\left(1 / g^{2}-\Pi_{X Y}^{\prime}\right) F_{\mu \nu}^{X} F_{\mu \nu}^{Y}$ (see Appendix A for details). ${ }^{6}$

To derive the value of $N$ in the present theory, we consider the $G$ sector as an analogue of the QCD. The standard analysis in large $N$ QCD [21] gives the relation between the pion decay constant, $f_{\pi}$, and the mass of the lowest spin- 1 resonance ( $\rho$ meson), $m_{\rho}$, as

$$
f_{\pi} \simeq \frac{\sqrt{N}}{4 \pi} m_{\rho} .
$$

In the present context, the pion decay constant, $f_{\pi}$, in the $G$ sector corresponds to the electroweak scale $v \simeq 175 \mathrm{GeV}$ and the mass of the $\rho$ meson, $m_{\rho}$, to the mass of the lowest KK excited gauge

\footnotetext{
${ }^{5}$ For $\kappa \equiv \tilde{g}^{2} N / 16 \pi^{2} \ll 1, f(\kappa)$ has an expansion $f(\kappa)=\sum_{0}^{\infty} c_{n} \kappa^{n}$, where $c_{n}=O(1)$. This is the domain where the perturbative $4 \mathrm{D}$ gauge theory description is appropriate. For $\kappa \gg 1, f(\kappa)$ again has an expansion but of the form $f(\kappa)=\sum_{0}^{\infty} c_{n}^{\prime} \kappa^{-n}$, where $c_{n}^{\prime}=O(1)$. This is the region where the theory is well described by a semi-classical gravitational theory, with higher order terms in the expansion corresponding to corrections from string theory. In the region $\kappa \simeq 1$, neither description is good and in the absence of the explicit string realization of the theory, we can only say that $f(\kappa)=O(1)$. In particular, for $\kappa \simeq N \simeq 1$, the theory does not admit any weakly coupled description and can only be described by strongly coupled $\left(g_{s} \simeq 1\right)$ string theory.

${ }^{6}$ Here we have taken matter in the $G$ sector, $\psi_{G}$, to be in the fundamental representation with the $O(1)$ number of "flavors". This may not be the case in our actual theory because the near conformality of the theory would require a large matter sector. However, for simplicity we will keep presenting our analysis for this simple matter sector, as it will give the correct relations between the physical quantities. We will come back to this point at the end of this subsection.
} 
boson $m_{1} \simeq(3 \pi / 4) k^{\prime}$. Then, rewriting $f_{\pi}$ and $m_{\rho}$ in Eq. (12) as $v$ and $m_{1}$ and using Eq. (8), we obtain the number of "colors", $N$, for $G:{ }^{7}$

$$
N \simeq 16 \pi^{2} \frac{v^{2}}{m_{1}^{2}} \simeq \frac{16 \pi^{2}}{\left(g_{L}^{2}+g_{R}^{2}\right) k} .
$$

Combined with Eq. (11), this equation tells us that the correction to the $S$ parameter becomes smaller if we make the first KK states heaver, i.e. $m_{1} / v$ larger, which can be attained by making either $g_{L}^{2} k$ or $g_{R}^{2} k$ larger. As was found in [6], this can be done by making $g_{R}^{2} / g_{L}^{2}$ larger even in the case of $1 / \tilde{g}_{L, 0}^{2} \simeq 1 / \tilde{g}_{Y, 0}^{2} \simeq 1 / 16 \pi^{2}$. An alternative possibility is to take all the bulk gauge couplings large, $1 / g_{L}^{2} \simeq 1 / g_{R}^{2} \simeq 1 / g_{X}^{2} \simeq M_{*} / 16 \pi^{3}$, and to assume that the $4 \mathrm{D}$ gauge couplings almost entirely come from the Planck-brane couplings.

Here we comment on the range of $N$ we are imagining. Because the $4 \mathrm{D}$ gauge couplings $g_{4 \mathrm{D}}$ receive contributions from the bulk gauge couplings, $g_{5 \mathrm{D}}$, we naturally expect that $1 / g_{4 \mathrm{D}}^{2} \gtrsim$ $\pi R / g_{5 \mathrm{D}}^{2}$ (see Eq. (8), for example). This relation can be written as $1 / g_{4 \mathrm{D}}^{2} \gtrsim \pi k R / g_{5 \mathrm{D}}^{2} k \simeq$ $\left(N / 16 \pi^{2}\right) \ln \left(k / k^{\prime}\right)$, using Eq. (13). Since the observed 4D gauge couplings are of order 1 , this gives constraints on $N: N \lesssim 16 \pi^{2} / \ln \left(k / k^{\prime}\right)$. This relation is understood in the $4 \mathrm{D}$ picture as the condition that the asymptotically non-free running of the $4 \mathrm{D}$ gauge couplings caused by the $G$ sector, $\left(N / 16 \pi^{2}\right) \ln \left(k / k^{\prime}\right)$, must not make the low-energy values of the gauge couplings, $g_{4 \mathrm{D}}$, too small. In any case, with $\ln \left(k / k^{\prime}\right) \simeq 30$, we obtain $N \lesssim 5$ so that we are not considering very large values of $N$ in the present context.

At leading order in $1 / N$, represented by the diagram in Fig. 2, the $T$ and $U$ parameters are not generated. This is because the $G$ sector respects the global custodial $S U(2)$ symmetry so that just inserting the $G$ dynamics, i.e. the gray disk, does not give $T$ or $U$ parameters [22]. Therefore, at this order, the electroweak oblique parameters receive contributions

$$
\begin{gathered}
S=c_{S} \frac{16 \pi}{\left(g_{L}^{2}+g_{R}^{2}\right) k}, \\
T=U=0,
\end{gathered}
$$

where $c_{S}$ is a coefficient of order unity.

What does this leading order contribution correspond to in the $5 \mathrm{D}$ picture? To see this, it is instructive to write Fig. 2 in a slightly deformed way as in Fig. 4. This diagram can be understood as the one in which the $W$ boson is transformed to some states made up of the constituents of the $G$ sector, and then goes back to $W$. Making a cut at the center of the figure (the dashed line denoted as $A$ in the figure) we find that these states are the bound states of

\footnotetext{
${ }^{7}$ Equivalently, the value of $N$ can be determined by the following argument. The diagram of Fig. 2 gives the squared masses for $W$ and $Z$ of order $\left(N / 16 \pi^{2}\right) m_{1}^{2}$, in the normalization where the gauge couplings appear in front of the gauge kinetic terms. Since these masses are $v^{2}$, we obtain $N \simeq 16 \pi^{2} v^{2} / m_{1}^{2}$.
} 


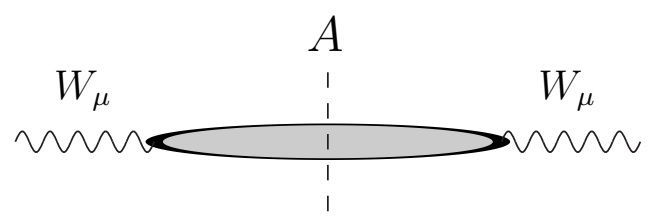

Figure 4: The diagram representing the mixing between the elementary $W$ boson and the composite $W$ states arising from the dynamics of $G$. Cutting the diagrams at $A$ gives the states which have the same quantum numbers and spin as $W$. Similar diagrams also exist for $W_{\mu}$ replaced by the gauge boson of $U(1)_{Y}, B_{\mu}$.

$G$ and have the same quantum numbers and spin as $W$. It is then clear that this represents mixings between the elementary $W$ boson and the excited $W$ bosons, the spin-1 bound states of $G$. Since these excited states correspond to KK states in the 5D picture, we learn that the contribution at leading order in $1 / N$ corresponds to the tree-level contribution in the 5D picture. In fact, by solving the masses and wavefunctions for the electroweak gauge bosons at tree level in 5D, we find that the contribution to $S$ takes the form of Eq. (14). In the absence of TeV-brane kinetic terms, the coefficient $c_{S}$ is given by $c_{S}=3 / 4$ (more detailed discussions including the $\mathrm{TeV}$-brane operators are given in the next subsection and in Appendix A). An interesting point here is that we can calculate the coefficient in Eq. (14), i.e. sum up the planar diagrams, and it gives the dominant contribution for sufficiently large $N$, i.e. for sufficiently small 5D gauge couplings $\left(g_{L}^{2}+g_{R}^{2}\right) k \ll 16 \pi^{2}$.

Before comparing with experiment, we discuss what happens at the next order. The next order contributions come at one loop in 5D, which corresponds to four different types of diagrams as shown in Fig. 5 (and diagrams with more insertions of the gray disk). The first one (Fig. 5a) is the loop of the elementary gauge bosons, the second one (Fig. 5b) represents the diagram at the next-to-leading order in $1 / N$, and the third and fourth ones (Fig. $5 \mathrm{c}, \mathrm{d}$ ) represent the ones with an additional loop of elementary fields to that of Fig. 2. The first diagram does not pick up effects of electroweak symmetry breaking so that it does not give contributions to $S, T$ or $U$. The third diagram (Fig. 5c) is also unimportant, since it gives only $S \simeq(N / \pi)\left(g^{2} / 16 \pi^{2}\right)$, which is always much smaller than the leading contribution of Eq. (11) (it is simply a higher order effect with the propagator of an internal elementary gauge boson corrected by the dynamics of $G)$. This diagram does not give contributions to the $T$ or $U$ parameters because generating them requires at least one additional insertion of the gray disk, to pick up the effect of custodial breaking encoded in the absence of elementary charged $S U(2)_{R}$ gauge bosons in the spectrum.

The second diagram (Fig. 5b) represents a correction coming entirely from the $G$ sector. Thus, as in the tree-level case, it does not give contributions to $T$ or $U$ parameter. Let us now 


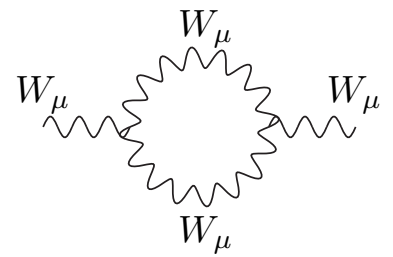

(a)

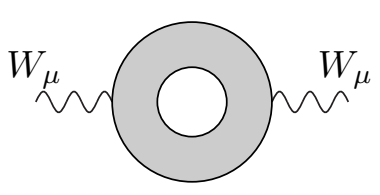

(b)

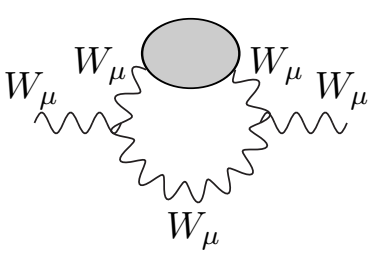

(c)

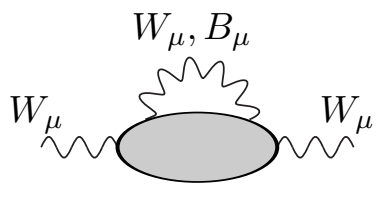

(d)

Figure 5: The diagrams with an elementary loop (a), with the $G$ effect at the next-to-leading order in $1 / N$ (b), and with an additional loop of elementary fields on top of the leading $G$ effect (c,d). For (a) and (c), similar diagrams using gauge 4-point vertices exist. For (b) and (d), there are also similar diagrams with $B_{\mu}$ on some of external lines.

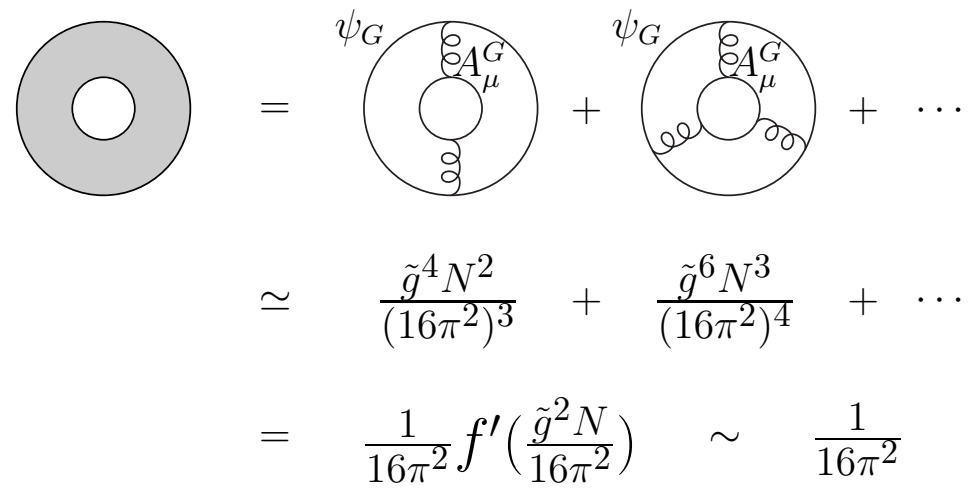

Figure 6: The diagrams represented by the gray annulus; $\psi_{G}$ and $A_{\mu}^{G}$ are matter and gauge fields of the $G$ sector. The contribution from this set of diagrams is of order $1 / 16 \pi^{2}$.

focus on the contribution to the $S$ parameter from this diagram. In a perturbative expansion, the disk with a hole is the sum of certain graphs as shown in Fig. 6. A similar reasoning as before implies that the contribution from this annulus is of order $1 / 16 \pi^{2}$, so that it gives a contribution to the $S$ parameter of order $S \simeq 1 / \pi$. It is then clear that, as long as $N \gtrsim 1$, the contribution from Fig. $5 \mathrm{~b}$ is at most comparable to the leading contribution of Eq. (11). Note that the condition $N \gtrsim 1$ is equivalent to the condition that the $5 \mathrm{D}$ gauge couplings are smaller than the value determined by NDA, $\left(g_{L}^{2}+g_{R}^{2}\right) \lesssim 16 \pi^{3} / M_{*}$, and the AdS curvature scale is smaller than the cutoff scale of the theory, $\pi k \lesssim M_{*}$ (see Eq. (13)), which we abide by throughout the paper. The precise value of $S$ coming from the diagram of Fig. 5 b depends on how we define the $S$ parameter. In particular, if we define our $S$ parameter to be the deviation from the 
standard model value, this contribution depends on the reference value for the physical Higgsboson mass, $m_{H}$, arbitrarily chosen to calculate the standard model contribution. However, this dependence on $m_{H}$ is not very important for later arguments in the paper. This is because, unless some calculable negative contribution to $S$ cancels the leading contribution of Eq. (14), the contribution from Fig. $5 \mathrm{~b}$ becomes important only in the region $N \simeq 1$, where all higher order corrections also become non-negligible and we are only able to say that the contribution to $S$ is of order $1 / \pi$.

The dominant contributions to the $T$ and $U$ parameters come from the diagram of Fig. $5 \mathrm{~d}$, whose size is estimated as $\left(N / 16 \pi^{2}\right)\left(g^{2} / 16 \pi^{2}\right)$, where $g \simeq 1$ represents generic electroweak gauge couplings. Since the natural scale of the $G$ sector is $m_{\rho}$, this gives contributions to the quantities $\Pi_{X X}(0)$ defined by $\mathcal{L}=-(1 / 2)\left(v^{2} / 2+\Pi_{X X}(0)\right) A_{\mu}^{X} A_{\mu}^{X}$ as $\Pi_{X X}(0) \simeq\left(N / 16 \pi^{2}\right)\left(g^{2} / 16 \pi^{2}\right) m_{\rho}^{2}$. Then, according to the definition of $T$, we have

$$
T \simeq \frac{16 \pi}{g^{2} v^{2}} \Pi_{X X}(0) \simeq \frac{1}{\pi},
$$

where we have used Eq. (12) with $f_{\pi}=v$. Again, the precise value of $T$, defined as the deviation from the standard model prediction, depends on the reference value for $m_{H}$ used to compute the standard model contribution. For the $U$ parameter, we note that the diagram of Fig. 5 d gives a contribution to $\Pi_{X X}^{\prime}$ of order $\left(N / 16 \pi^{2}\right)\left(g^{2} / 16 \pi^{2}\right)$. Then, from the definition of $U$, we obtain

$$
U \simeq 16 \pi \Pi_{X X}^{\prime} \simeq \frac{g^{2} v^{2}}{m_{1}^{2}} T .
$$

This shows that as long as $\left(g v / m_{1}\right)^{2} \ll 1$, as in our case, the contribution to the $U$ parameter is negligible.

By deforming the diagrams of Fig. 5 as in the way we deformed Fig. 2 to Fig. 4, we can easily see that these diagrams actually correspond in the 5D picture to the one-loop diagrams in which the KK gauge bosons circulate in the loop. The reason why these diagrams can give only subdominant contributions to $S$ is then clear because the size of the loop diagrams is always smaller than that of the tree-level effects, unless the quantity is first generated at the loop level as in the case of the $T$ and $U$ parameters. (Remember that $N \gtrsim 1$ corresponds to the condition that the $5 \mathrm{D}$ gauge couplings, $g_{5 \mathrm{D}}$, are smaller than the value given by NDA: $g_{5 \mathrm{D}}^{2} \lesssim 16 \pi^{3} / M_{*}$.) From the above 4D analysis, we know that the 5D loop contributions to $S$ and $T$, given by $S \simeq T \simeq 1 / \pi$, do not depend on $N$, i.e. the mass scale of the KK excitations. In fact, in the $5 \mathrm{D}$ theory we find that the 3-point couplings involving a lowest-mode (our $W$ and $Z$ ) and two KK gauge bosons scale as $1 / \sqrt{N} \propto m_{1} / v$. Thus they cancel the mass suppression arising from the KK gauge-boson propagators when we calculate $S$ and $T$, making these contributions non-decoupling. 
We close this subsection with a final important remark. In the above discussion, we have presented our analysis assuming that matter fields in the $G$ sector, $\psi_{G}$, transform as the fundamental representation under $G$. We have also implicitly assumed that the number of these fields are of $O(1)$ and not of $O(N)$. These assumptions, however, will most likely be violated in the present theory because it must be nearly conformal above the scale $\Lambda \approx k^{\prime}$, which requires large representations or a large number of matter fields to make the beta function nearly vanishing. Nevertheless, this will not change any of our physical conclusions described here and below. Let us, for example, consider the case where the matter sector consists of $O(1)$ number of $\psi_{G}$ 's that transform as the adjoint representation under $G$. In this case the gray disks in Figs. $2-4,5$ c and $5 \mathrm{~d}$ are replaced by gray spheres, which give contributions of order $N^{2} / 16 \pi^{2}$, and consequently $N$ in Eqs. $(11,12,13)$ must be replaced by $N^{2}$. Similarly, the gray annuli in Figs. 5b and 6 are replaced by gray tori. However, these replacements do not change any of the relations between the physical quantities - it simply says that the quantity called $N$ before must actually be identified as $N^{2}$. (Note that not all of the $N^{\prime}$ 's must be replaced by $N^{2}$. For example, the expansion in Figs. 3 and 6 are given by $\left(N^{2} / 16 \pi^{2}\right) f\left(\tilde{g}^{2} N / 16 \pi^{2}\right)$ and $\left(1 / 16 \pi^{2}\right) f^{\prime}\left(\tilde{g}^{2} N / 16 \pi^{2}\right)$, respectively, and not $\left(N^{2} / 16 \pi^{2}\right) f\left(\tilde{g}^{2} N^{2} / 16 \pi^{2}\right)$ and $\left(1 / 16 \pi^{2}\right) f^{\prime}\left(\tilde{g}^{2} N^{2} / 16 \pi^{2}\right)$. Basically, $N$ appearing in $\kappa \equiv \tilde{g}^{2} N / 16 \pi^{2}$ is not replaced by $N^{2}$.) One consequence of considering adjoint matter is that the corresponding gravitational theory now seems to be closed string theory, because the sphere and torus do not have any edge identified as the endpoint of strings (for another implication, on an understanding of the fermionic KK towers, see footnote 8). The situation is similar in the case of $O(N)$ number of $\psi_{G}$ 's transforming as the fundamental representation: the quantity called $N$ should be identified as $N^{2}$, although the disks and annuli in this case are not replaced by other objects and the corresponding gravitational theory is still an open string theory. In the rest of the paper, we keep using $N$ as it appeared in the heuristic presentation in this subsection, as it will not change any of the physical results. The reader who wants a more precise picture, however, should understand it appropriately as the square of the number of "colors" of the gauge group $G$.

\subsection{Comparison with experimental data: $S$ parameter}

Current experimental data already give strong constraints on possible new physics at the $\mathrm{TeV}$ scale. For example, the absence of FCNCs other than those arising from the standard model strongly constrains the flavor structure for the $\mathrm{TeV}$ physics. Here we concentrate on the constraints arising from the precision electroweak data, especially those on $S$ and $T$ oblique parameters. The issue of flavor changing processes will be discussed in the next section.

We first note that our $S$ and $T$ parameters are defined as the deviations from the standard 
model values. The standard model contributions to the vacuum polarizations are calculated once the mass of the physical Higgs boson, $m_{H}$, is specified. On the other hand, in our theory there is no Higgs boson in the spectrum so that the contributions to the vacuum polarizations do not depend on such parameter. This means that $S$ and $T$, defined as the differences between the vacuum polarizations in our theory and those in the standard model, depend on $m_{H}$, which is arbitrary chosen to calculate the standard model contribution. Specifically, $S$ and $T$ arising from the diagrams of Figs. $5 \mathrm{~b}$ and $5 \mathrm{~d}$ depend on the parameter $m_{H}$. Of course, this dependence on $m_{H}$ is not physical - the experimental constraints on $S$ and $T$ also depend on $m_{H}$, and the physical constraints on (the $G$ sector of) our model do not depend on the arbitrary parameter $m_{H}$. Treating this issue correctly would become important when we aim to make a precise comparison between the predictions of the theory and experiments. However, we do not need such a precision for the purpose here, as we do not attempt to make the comparison between the theory and experiment at the level of 5D one-loop contributions. Rather, we discuss general implications of the results in the previous subsection, focusing on the large leading-order contribution.

We therefore regard that the theory is successful if it gives sufficiently small values of $S$ and $T$. Specifically, we here take a somewhat conservative criterion $S, T \lesssim 1 / \pi$, and consider whether the contribution from the gauge sector derived in the previous subsection satisfies it. Below, we will explicitly see that the minimal theory with a perturbative 5D energy region fails to pass this test, which implies that either the extension of the model or the deviation from the perturbative 5D picture is necessary for the theory to be viable. As we will see in the next section, our conclusion is not changed by including contributions from the matter sector.

With our weak criterion $S, T \lesssim 1 / \pi$, only the dangerous contribution is the leading-order contribution to the $S$ parameter given in Eq. (11) or Eq. (14). These equations imply that, if the coefficient $c_{S}$ is order 1 , we need to go to the parameter region $N \simeq 1$, which requires that the $5 \mathrm{D}$ theory is strongly coupled already at the scale of the lowest excitation. To see the situation more quantitatively, however, we have to calculate the coefficient $c_{S}$. This can be done in $5 \mathrm{D}$ by solving the equations of motion for the gauge fields at tree level.

In order to analyze the most general situation, we add the following gauge kinetic terms localized on the $\mathrm{TeV}$ brane

$\mathcal{S}=\int d^{4} x \int d y \sqrt{-g_{\text {ind }}} \delta(y-\pi R)\left[\sum_{a=1}^{3}\left\{-\frac{Z_{L}}{4} F_{\mu \nu}^{L a} F_{\mu \nu}^{L a}-\frac{Z_{R}}{4} F_{\mu \nu}^{R a} F_{\mu \nu}^{R a}-\frac{Z_{M}}{2} F_{\mu \nu}^{L a} F_{\mu \nu}^{R a}\right\}-\frac{Z_{X}}{4} F_{\mu \nu}^{X} F_{\mu \nu}^{X}\right]$,

in addition to the bulk and Planck-brane localized gauge kinetic terms, Eq. (5). We can now compute the coefficient $c_{S}$ as a function of the parameters of the theory, $1 / g_{L}^{2}, 1 / g_{R}^{2}, 1 / g_{X}^{2}, 1 / \tilde{g}_{L}^{2}$, $1 / \tilde{g}_{Y}^{2}, Z_{L}, Z_{R}, Z_{M}$, and $Z_{X}$. The detailed calculation is given in Appendix A, and the result can 
be summarized as

$$
S=c_{S} \frac{N}{\pi}
$$

where

$$
N=\frac{16 \pi^{2}}{\left(g_{L}^{2}+g_{R}^{2}\right) k}, \quad c_{S}=\frac{3}{4}+\frac{g_{L}^{2} k \cdot g_{R}^{2} k}{\left(g_{L}^{2}+g_{R}^{2}\right) k}\left(Z_{L}+Z_{R}+Z_{M}\right) .
$$

In the absence of the TeV-brane operators, $Z_{L}=Z_{R}=Z_{M}=0$, we find that $c_{S}=3 / 4$ and $N$ must actually be small, $N \simeq 1$, for the theory to be viable. The smallest value of $N$ is obtained at the largest values for the bulk gauge couplings. Suppose that one of $g_{L}$ or $g_{R}$ becomes strongly coupled at the cutoff scale of the 5D theory, $M_{*}$. This implies that at least one of $g_{L}$ or $g_{R}$ is as large as $g_{L, R}^{2} \simeq 16 \pi^{3} / M_{*}$, and thus we obtain $N \simeq M_{*} /(\pi k)$. Therefore, we find that the contribution to $S$ becomes sufficiently small only when the $5 \mathrm{D}$ cutoff scale is lowered down to the scale of AdS curvature (i.e. the IR cutoff scale, $M_{*}^{\prime}$, is lowered to the scale close to the mass of the first $\mathrm{KK}$ resonance, $m_{1} \simeq \pi k^{\prime}$.

At first sight, the above conclusion seems to change if we introduce the TeV-brane gauge kinetic terms, $Z_{L}, Z_{R}, Z_{M} \neq 0$, because of the second term in the equation for $c_{S}$ in Eq. (20). A careful study, however, shows that the conclusion actually persists even in the presence of the TeV-brane terms. To see this, we first rewrite $Z_{L}, Z_{R}$ and $Z_{M}$ as $Z_{L} \equiv \delta_{L} / 16 \pi^{2}, Z_{R} \equiv \delta_{R} / 16 \pi^{2}$ and $Z_{M} \equiv \delta_{M} / 16 \pi^{2}$, respectively. The NDA values for these coefficients are then represented as $\delta_{L}, \delta_{R}, \delta_{M}=O(1)$. An important point here is that these parameters cannot take large negative values, because it would lead to a ghost below the IR cutoff scale, $M_{*}^{\prime}$. We thus have constraints $\delta_{L}, \delta_{R}, \delta_{M} \gtrsim-1$ from the consistency of the theory, which gives a strong restriction on the possibility that the second term in the expression of $c_{S}$ cancels the first term and gives smaller values of $c_{S}$ (and thus allows larger values of $N$ ). The second term of $c_{S}$ in Eq. (20) becomes most negative when at least one of $g_{L}$ or $g_{R}$ takes the largest value, in which case

$$
c_{S} \simeq \frac{3}{4}+\frac{\pi k}{M_{*}}\left(\delta_{L}+\delta_{R}+\delta_{M}\right)
$$

We thus find that $c_{S}$ can be much smaller than $O(1)$ only when the two scales $M_{*}$ and $\pi k$ are close. In fact, Eq. (21) suggests that the effect encoded in the TeV-brane kinetic terms should be regarded as "stringy corrections", i.e. the higher order effect in the $1 / \kappa$ expansion.

The argument described above explicitly shows that the minimal theory with a large perturbative $5 \mathrm{D}$ energy interval, $M_{*} \gg \pi k$, fails to comply with precision electroweak data, because then we have $c_{S} \gtrsim 1$ and $N \gg 1$. There are then two possibilities to make the theory viable. The first one is to extend the minimal model to include a new sector that gives a negative contribution to $S$ and cancels the leading gauge contribution of Eq. (19). Such a contribution may (effectively) arise from additional matter fields (localized to the TeV brane) [23], additional 
gauge bosons [24] or, perhaps, even from the physics associated with the radion field [25] in which case the extension of the model may not actually be needed. In this case, the 5D theory can be perturbative up to the cutoff scale $M_{*}$ which is parametrically higher than the AdS curvature scale $\pi k$. This means that the strongly-coupled $G$ sector that breaks electroweak symmetry has a weak coupling description over a certain energy interval above the mass of the lowest excitation, $\pi k^{\prime}$, up to some higher energy scale, $M_{*}^{\prime}$. This type of theory would then allow precise computations of electroweak corrections, for example $S, T$ and $U$ parameters generated at the 5D loop level (although for $S$ there are intrinsic uncertainties of the same order arising at tree level from operators on the TeV brane). The unitarity of the longitudinal $W W$ scattering amplitudes is recovered by the presence of the electroweak KK gauge bosons, instead of the Higgs boson $[7,26]$. The required cancellation to attain these is of order $1 / N \simeq \pi k / M_{*}$. Thus, in order to have a reasonable energy interval where the theory has a weak coupling description, say $M_{*} /(\pi k) \simeq 5$, we only have to invoke the cancellation of order $20 \%$.

The second possibility is to give up a weak coupling description of the theory; specifically, we take $M_{*} \simeq \pi k$. In this case the mass of the first excited mode, $m_{1} \simeq \pi k^{\prime}$, is close to the scale where the theory becomes truly strongly coupled, $M_{*}^{\prime}$. There is no energy interval where the theory admits a weak coupling description, and electroweak radiative corrections cannot be reliably computed. Nevertheless, NDA suggests that in this parameter region the corrections to $S$ and $T$ are both of order $1 / \pi$. Therefore, it does not seem so unnatural that these corrections in fact do satisfy the constraints from precision electroweak measurements. The worry, of course, is that because the cutoff scale is close to the first KK mass, the 5D field theoretic description of the theory may not make much sense. For instance, if the 5D Planck scale $M_{5}$ is taken close to the 5D cutoff scale, as in the usual case, the background AdS solution itself will receive large quantum gravitational corrections and our entire treatment will become unreliable. However, the 5D Planck scale, $M_{5}$, may be parametrically larger than the cutoff scale, $M_{*}$. The argument based on the locality in 5D may also persist even for $M_{*} \simeq \pi k$, as the proper distance for the fifth dimension, $\pi R$, is still larger than the cutoff length, $1 / M_{*}$. Here we do not try to make further arguments on the viability of this parameter region. A more solid treatment of this region will probably require a string theoretic construction of the theory.

Having the above two possibilities in mind, we will discuss further phenomenological issues of the theory in the next section. These include flavor violation, top quark phenomenology, and contributions of the matter sector to the electroweak oblique parameters. 


\section{Fermion Sector and Its Phenomenology}

In this section we discuss the fermion sector of the model described in section 3 . We focus most of our discussions on the third generation quarks since they are most severely constrained by experiments. However, some of our analyses, for instance those for mass eigenvalues and flavor violation, are also applicable to the first two generation quarks and leptons. A related study on the issue of fermion masses can be found in [27].

\subsection{Basic structure}

Let us start by summarizing the structure of the fermion sector of the model given in section 3 . A single generation of the quark sector consists of the following fermion content:

$$
\begin{aligned}
& q(\mathbf{2}, \mathbf{1}, 1 / 6)=\left(\begin{array}{c}
u \\
d
\end{array}\right), \quad \psi_{\bar{u}}(\mathbf{1}, \mathbf{2},-1 / 6)=\left(\begin{array}{c}
\bar{D} \\
\bar{u}
\end{array}\right), \quad \psi_{\bar{d}}(\mathbf{1}, \mathbf{2},-1 / 6)=\left(\begin{array}{c}
\bar{d} \\
\bar{U}
\end{array}\right), \\
& q^{c}\left(\mathbf{2}^{*}, \mathbf{1},-1 / 6\right)=\left(\begin{array}{c}
u^{c} \\
d^{c}
\end{array}\right), \quad \psi_{\bar{u}}^{c}\left(\mathbf{1}, \mathbf{2}^{*}, 1 / 6\right)=\left(\begin{array}{c}
\bar{D}^{c} \\
\bar{u}^{c}
\end{array}\right), \quad \psi_{\bar{d}}^{c}\left(\mathbf{1}, \mathbf{2}^{*}, 1 / 6\right)=\left(\begin{array}{c}
\bar{d}^{c} \\
\bar{U}^{c}
\end{array}\right) .
\end{aligned}
$$

Here $q, \psi_{\bar{u}}, \psi_{\bar{d}}, q^{c}, \psi_{\bar{u}}^{c}$ and $\psi_{\bar{d}}^{c}$ represent (left-handed) Weyl fermions; $q$ and $q^{c}$ form a single $5 \mathrm{D}$ (Dirac) fermion, and the same is true for $\psi_{\bar{u}}$ and $\psi_{\bar{u}}^{c}$, and for $\psi_{\bar{d}}$ and $\psi_{\bar{d}}^{c}$. The numbers in parentheses on the left-hand side of the equations represent the quantum numbers under $S U(2)_{L} \times S U(2)_{R} \times U(1)_{X}$.

Under the orbifold boundary conditions the zero modes for the conjugate fields are projected out, so that the fields in the lower line of Eq. (22) do not have zero modes. The zero modes for $\bar{U}$ and $\bar{D}$ also get masses by marrying with the Planck-brane fields $\psi_{\bar{u}}^{\prime}$ and $\psi_{\bar{d}}^{\prime}$ (see discussions below Eq. (6)). Therefore, before turning on the effect of electroweak symmetry breaking, the three Weyl-fermion fields $q, \bar{u}$ and $\bar{d}$ are massless. These fields have the quantum numbers $(\mathbf{2}, 1 / 6)$, $(\mathbf{1},-2 / 3)$ and $(\mathbf{1}, 1 / 3)$ under $S U(2)_{L} \times U(1)_{Y}$ and are identified as the quarks in the standard model. The fermion KK towers consist of all the fields listed in Eq. (22).

Once the effect of electroweak symmetry breaking is introduced through the operators in Eq. (7), the standard-model quarks receive masses, which depend on the bulk mass parameters $c$ for $q, \psi_{\bar{u}}, \psi_{\bar{d}}$ as well as the TeV-brane couplings, $y_{u}$ and $y_{d}$. These masses, together with the KK tower mass spectrum, are worked out in Appendix B. They are determined by the condition

$$
\begin{aligned}
& \left(J_{c_{L}-\frac{1}{2}}\left(\frac{m}{k^{\prime}}\right)-\frac{J_{c_{L}-\frac{1}{2}}\left(\frac{m}{k}\right)}{Y_{c_{L}-\frac{1}{2}}\left(\frac{m}{k}\right)} Y_{c_{L}-\frac{1}{2}}\left(\frac{m}{k^{\prime}}\right)\right)\left(J_{c_{R}-\frac{1}{2}}\left(\frac{m}{k^{\prime}}\right)-\frac{J_{c_{R}-\frac{1}{2}}\left(\frac{m}{k}\right)}{Y_{c_{R}-\frac{1}{2}}\left(\frac{m}{k}\right)} Y_{c_{R}-\frac{1}{2}}\left(\frac{m}{k^{\prime}}\right)\right) \\
& -|\lambda|^{2}\left(J_{c_{L}+\frac{1}{2}}\left(\frac{m}{k^{\prime}}\right)-\frac{J_{c_{L}-\frac{1}{2}}\left(\frac{m}{k}\right)}{Y_{c_{L}-\frac{1}{2}}\left(\frac{m}{k}\right)} Y_{c_{L}+\frac{1}{2}}\left(\frac{m}{k^{\prime}}\right)\right)\left(J_{c_{R}+\frac{1}{2}}\left(\frac{m}{k^{\prime}}\right)-\frac{J_{c_{R}-\frac{1}{2}}\left(\frac{m}{k}\right)}{Y_{c_{R}-\frac{1}{2}}\left(\frac{m}{k}\right)} Y_{c_{R}+\frac{1}{2}}\left(\frac{m}{k^{\prime}}\right)\right)=0,
\end{aligned}
$$


where $m$ represents the mass eigenvalues: the masses for our quarks and the KK towers are given as the solutions to this equation. Here $J_{\nu}(x)$ and $Y_{\nu}(x)$ are Bessel functions, $c_{L}$ and $c_{R}$ are the bulk masses for the left-handed and right-handed fermions, and $\lambda$ represents the size of the TeV-brane operators. For example, if we want to know the mass eigenvalues of the up-type quark KK tower, we substitute $c_{L}=c_{q}, c_{R}=c_{\psi_{\bar{u}}}$ and $\lambda=y_{u} v_{H}$ in Eq. (23) and solve. In the case of the down-type quark, we use $c_{L}=c_{q}, c_{R}=c_{\psi_{\bar{d}}}$ and $\lambda=y_{d} v_{H}$. For the leptons, $c_{L}=c_{l}$, $c_{R}=c_{\psi_{\bar{e}}}$ and $\lambda=y_{e} v_{H}$.

For the first two generation quarks (and leptons), we choose $c_{L}$ and $c_{R}$ sufficiently larger than $1 / 2$. In this case the lightest mass eigenvalues, i.e. the masses of our quarks and leptons, are

suppressed by a large factor, $m \sim k^{\prime}\left(k^{\prime} / k\right)^{c_{L}+c_{R}-1}$, explaining the hierarchy among quark and lepton masses [17]. Because we do not want to have such a suppression for the third generation quarks, especially for the top quark, we choose $c_{q}$ and $c_{\psi_{\bar{u}}}$ for the third generation to be smaller than $1 / 2$. The third generation $c_{\psi_{\bar{d}}}$ is taken to be larger than but close to $1 / 2$ so that the bottom quark mass is not too suppressed. More detailed discussions on phenomenology of the third generation sector, including the mass spectrum of the top-quark KK tower, appear in the following subsections.

\subsection{Constraints from flavor violation}

Since the electroweak gauge symmetry is broken by boundary conditions or a large VEV of the brane-localized field $H$, the wavefunctions of the $W$ and $Z$ bosons in our theory are not flat in the extra dimension. This generically introduces non-universality of the electroweak gauge couplings depending on the bulk fermion mass parameters, because the 4D gauge couplings are obtained by convolving the wavefunctions of the corresponding fermion with the gauge boson, which are not universal for fermions having different values of the bulk masses. To estimate the size of this non-universality, we first consider the theory in the $4 \mathrm{D}$ picture. In this picture the non-universality for the electroweak gauge couplings is caused by the diagram as shown in Fig. 7a. Here, the solid external lines are fermion fields and the wavy external line is the electroweak gauge boson. The gray disk at the center represents the dynamics of the $G$ sector.

What is the coupling of the external fermion lines (elementary fermion fields) to the $G$ sector? In the $4 \mathrm{D}$ picture, an elementary fermion field $\psi$ couples to the $G$ sector through the interaction like $\mathcal{L}_{4 \mathrm{D}} \sim \psi \mathcal{O}_{\psi}$, where $\mathcal{O}_{\psi}$ is an operator which consists of fields in the $G$ sector and has the quantum numbers of $\psi$ conjugate. The dimension of this operator is related to the bulk mass $c$ of the 5D field corresponding to $\psi$. For $c \geq-1 / 2$ it is given by $\left[\mathcal{O}_{\psi}\right]=c+2$ [28]. Therefore, in terms of the canonically normalized field $\psi$, the coupling of $\psi$ and the $G$ sector can be written 


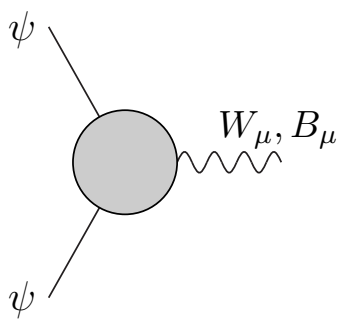

(a)

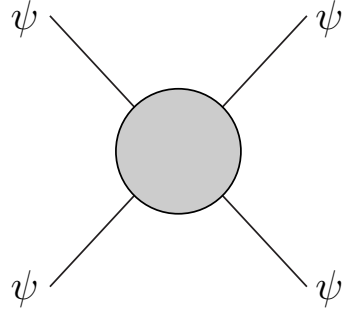

(b)

Figure 7: Diagrams giving flavor non-universal gauge interactions (a) and four-fermion operators (b). The gray disks represent the dynamics of the $G$ sector.

as

$$
\mathcal{L}_{4 \mathrm{D}}=\frac{\eta}{k^{c-1 / 2}} \psi \mathcal{O}_{\psi},
$$

where $\eta$ is a dimensionless coupling constant. For $c>1 / 2$ this is an irrelevant operator and the coupling $\eta$ is of order one. For $c<1 / 2$, the interaction in Eq. (24) is relevant, and the coupling $\eta$ runs with energy as $\eta(\mu) \sim(k / \mu)^{c-1 / 2}$, which implies that $\eta / k^{c-1 / 2}$ provides an order-one insertion at any given energy [4]. For $c=1 / 2$ the operator is marginal and $\eta$ is given by $\eta \sim 1 /\left(\ln \left(k / k^{\prime}\right)\right)^{1 / 2}$ at the electroweak scale. For $c$ larger than but close to $1 / 2$, $\eta \simeq\left((2 c-1) /\left(1-\left(k^{\prime} / k\right)^{2 c-1}\right)\right)^{1 / 2}$.

We can now estimate the non-universality arising from the diagram of Fig. 7a. For $c>1 / 2$ the fermion field is attached to the gray disk with the factor $\eta / k^{c-1 / 2}$. The gray disk contribute as $N / 16 \pi^{2} \sim 1 /\left(g_{L}^{2}+g_{R}^{2}\right) k{ }^{8}$ Multiplying these factors and supplying the dimension by $k^{\prime}$ then leads to the non-universality of the gauge coupling $\delta g$ :

$$
\frac{\delta g}{g}=a \frac{\eta^{2} k^{2 c-1}}{\left(g_{L}^{2}+g_{R}^{2}\right) k^{2 c}} .
$$

where $a$ is an $O(1)$ constant. In fact, this parametric dependence can be recovered in the 5D calculation. Defining the non-universality $\delta g$ as the deviation of the gauge coupling from the case

\footnotetext{
${ }^{8}$ This estimate may be justified by the following argument. The fact that the $4 \mathrm{D}$ gauge couplings receive contributions from the $G$ sector proportional to $N$ implies that the $G$ matter charged under $S U(2)_{L} \times U(1)_{Y}$ is in fundamental representations of $G$. Then, the fields circulating on the edge of the disk must be fundamental representations of $G$ (the edge between the two external fermion lines is a scalar), and the standard counting in large $N$ for the group-theoretical factor gives $N / 16 \pi^{2}$. An alternative possibility is that matter in the $G$ sector, $\psi_{G}$, is in the adjoint representation under $G$ (see the last paragraph of section 4.1). In this case, the gray disk becomes a gray sphere and contributions from the $G$ sector to the $4 \mathrm{D}$ gauge couplings are proportional to $N^{2}$. The $N$ power counting perfectly works just by replacing $N$ by $N^{2}$. The adjoint matter also provides an understanding of the KK towers of the fermion fields as bound states of $\psi_{G}$ and $A_{\mu}^{G}$ (the gauge bosons of $G$ ). A fundamental scalar field is not necessary in this case.
} 
of Planck-brane localized fermions, we obtain it by convolving the wavefunctions of the matter zero mode and the electroweak gauge boson. We find that $a \simeq 1-10$ that depends quite weakly on $c$ (the $a$ here contains the possible effect from brane couplings (see Eq. (26) below), which arises from the fact that our $W$ and $Z$ bosons are mixtures of elementary and composite states). This constitute a small non-universality, and the constraints from flavor violating processes for the first two generation quarks (as well as leptons) are evaded relatively easily [6], especially when $g_{R}$ is large, i.e. the mass of the first KK gauge boson becomes large. Similarly, nonuniversal four-fermion operators generated by the exchange of the KK gauge bosons, represented by the diagram of Fig. 7b, are also quite small for the first two generations. They are given by $\delta G_{F} \simeq \eta^{4}\left(k^{\prime} / k\right)^{4 c-2} /\left(\left(g_{L}^{2}+g_{R}^{2}\right) k k^{\prime 2}\right)$, where $\delta G_{F}$ represents the coefficients of the flavor violating four-fermion operators obtained after integrating out the KK gauge bosons. ${ }^{9}$

For the third generation quarks, $c_{q}$ and $c_{\psi_{\bar{u}}}$ must be smaller than $1 / 2$ in order to give a large enough mass to the top quark. The value of $c_{q}$ is then constrained by the flavor violating coupling of the left-handed $b$ quark to the $Z$ boson. Performing a full $5 \mathrm{D}$ calculation, we find that the relevant flavor violation is parameterized by

$$
\frac{\delta g_{L}^{b}}{g_{L}^{b}}=\frac{f}{\left(g_{L}^{2}+g_{R}^{2}\right) k}\left(\frac{g_{L}^{2}}{\pi R g^{2}}\right),
$$

where $g_{L}^{b}$ is the coupling of the left-handed bottom quark to the $Z$ boson, and $f=f\left(c_{L}, c_{R}, \lambda\right)$ is a function of $c_{L}=c_{q}, c_{R}=c_{\psi_{\bar{d}}}$ and $\lambda=y_{d} H$. The last factor captures the dependence of $\delta g_{L}^{b} / g_{L}^{b}$ on the brane couplings, becoming 1 in their absence (see Eq. (8)). For $c_{R}>1 / 2$ and $\lambda \lesssim 1$, the coefficient $f$ effectively depends only on $c_{L}$. The dependence is roughly given by

$$
f\left(c_{L}\right) \approx-a^{\prime} \eta^{2}=-a^{\prime} \frac{1-2 c_{L}}{1-\left(k^{\prime} / k\right)^{1-2 c_{L}}},
$$

where the dependence of $a^{\prime}$ on $c_{L}$ is rather weak (for instance, $6 \lesssim a^{\prime} \lesssim 7$ in the parameter region we are interested, $0.3 \lesssim c_{L} \lesssim 0.5$ ). In Fig. 8 we plot $f$ as a function of $c_{L}$, calculated in the 5D picture. The $Z \rightarrow b \bar{b}$ decay constrains the quantity $\delta g_{L}^{b} / g_{L}^{b}$ to be less than a percent level. In our theory, $\left(g_{L}^{2}+g_{R}^{2}\right) k \simeq 16 \pi^{2} / N$, and in the case that the brane couplings are given by Eqs. (10) with $1 / \tilde{g}_{L, 0}^{2} \simeq 1 / \tilde{g}_{Y, 0}^{2} \simeq 1 / 16 \pi^{2}$, we find from Eq. (26) and Fig. 8 that $c_{L}$ must satisfy $c_{L} \gtrsim 0.3,0.44$ and 0.47 for $N \simeq 1,3$ and 5 , respectively. Therefore, to avoid having to fine-tune the parameter $c_{L}$ to be very close to $1 / 2$, smaller values of $N$ are preferred. This points in the same direction as the leading-order $S$ parameter constraint, although the constraint from $Z \rightarrow b \bar{b}$ is weaker and allows a reasonable energy interval for the weakly coupled $5 \mathrm{D}$ description, e.g. $N \simeq 3$. In the

\footnotetext{
${ }^{9}$ Diagrams similar to Figs. 7a and $7 \mathrm{~b}$ but with $\psi$ 's replaced by gauge fields give corrections to the gauge three-point and four-point interactions, $Z W W, W W W W$ and $Z Z W W$. These corrections are of order $\delta g / g \simeq$ $\left(N / 16 \pi^{2}\right) g^{2} \simeq g^{2} v^{2} / m_{1}^{2}$.
} 


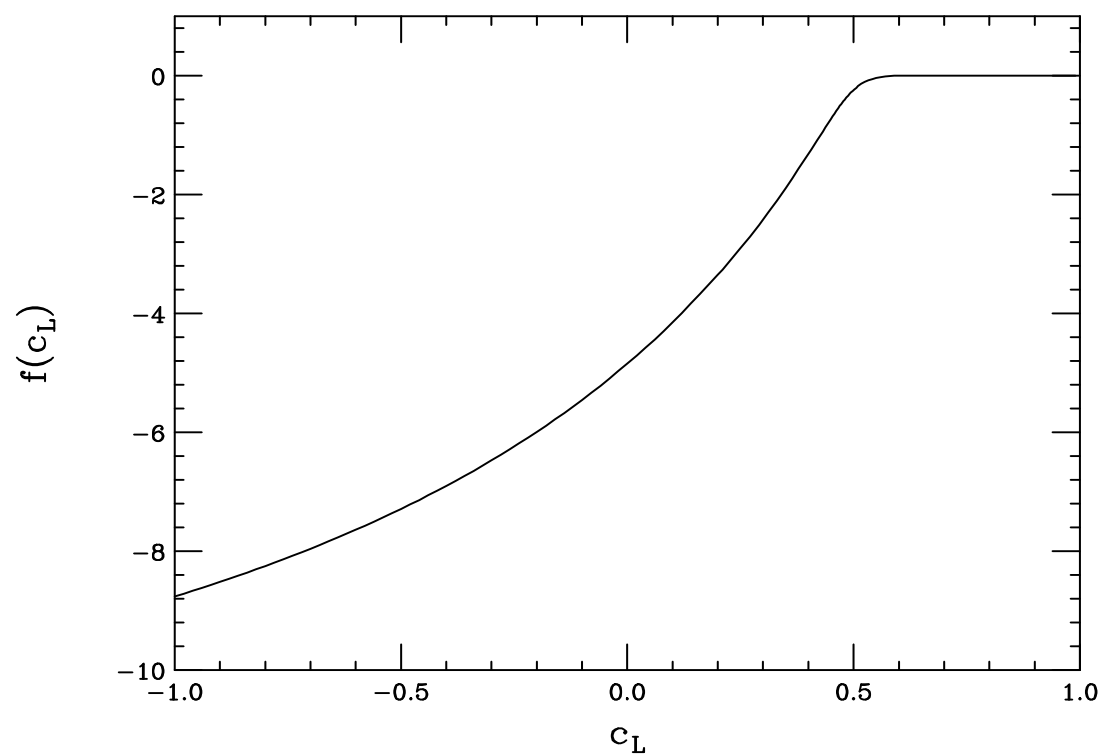

Figure 8: The function $f$ defined in Eq. (26), which determines the deviation of $g_{L}^{b}$ from its standard model value.

case of strong bulk gauge couplings, Eq. (26) gives about one percent deviation for $c_{L} \simeq 0.49$ for $1 / g_{L, R, X}^{2} \simeq M_{*} / 16 \pi^{3}$. Smaller values of $c_{L}$, however, are possible if $g_{L}$ takes somewhat smaller values, e.g. $g_{L}^{2} \lesssim 8 \pi^{3} / M_{*}$, or there is some cancellation from unknown strong coupling dynamics.

\subsection{Top quark phenomenology}

Obtaining a large enough mass for the top quark is a non-trivial issue in any theory with dynamical electroweak symmetry breaking. In this subsection we discuss the spectrum of the top quark and its KK tower. We also address the resulting top-quark phenomenology.

The relevant parameters for the top-quark sector are $c_{L}=c_{q}, c_{R}=c_{\psi_{\bar{u}}}$ and $\lambda=y_{u} H$ for the third generation. As seen in the previous subsection, the parameter $c_{L}$ must be close to $1 / 2$ to avoid the conflict with the observed $Z \rightarrow b \bar{b}$ decay rate. The parameter $c_{R}$ is less constrained and can take much smaller values. For fixed values of $c_{L}$ and $c_{R}$, the spectrum of the top KK tower shows the following behavior as a function of $\lambda$. For $\lambda=0$, the spectrum consists of two decoupled towers for the 5D fields $u$ and $\bar{u}$. Each tower has a zero mode, which is a Weyl fermion, and a tower of Dirac fermions; the towers for $u$ and $\bar{u}$ have identical masses for $c_{L}=c_{R}$, but in general have slightly different masses for $c_{L} \neq c_{R}$. Therefore, the overall spectrum for $\lambda=0$ can be described as follows: there is a Dirac fermion at the massless level, which consists of two Weyl-fermion zero modes of $u$ and $\bar{u}$, and the KK tower has two Dirac fermions at each 


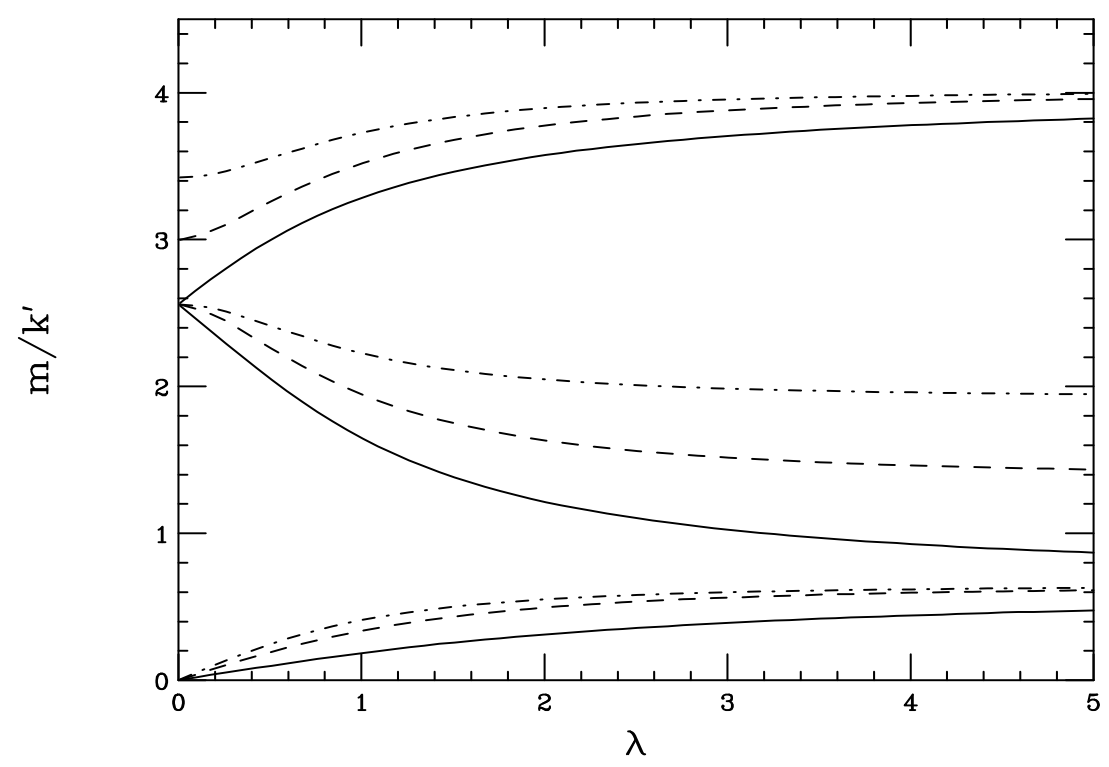

Figure 9: The spectra for the top KK tower. The horizontal axis is $\lambda=y_{u} H$, and the vertical axis is the masses in units of $k^{\prime}$. The solid, dashed and dot-dashed lines are for $\left(c_{L}, c_{R}\right)=(0.4,0.4)$, $(0.4,0.1)$ and $(0.4,-0.2)$, respectively.

level, arising from $u$ and $\bar{u}$, whose masses are degenerate for $c_{L}=c_{R}$ but not in general. When we turn on $\lambda$ by a small amount $(\lambda \ll 1)$, the Dirac fermion that is massless for $\lambda=0$ receives a mass proportional to $\lambda$. Meanwhile, the two Dirac fermions at each level become increasingly split: the lightest of the two becomes lighter and the heaviest becomes heavier. For very large $\lambda$ $(\lambda \gg 1)$, the masses for all the states become constant. In particular, the mass of the formerly massless state approaches to a constant value of order $k^{\prime}$. The lightest of the first excited states becomes close to this state in mass. They become degenerate at $\lambda \rightarrow \infty$ for $c_{L}=c_{R}$, but not for $c_{L} \neq c_{R}$.

The behavior of the mass eigenvalues described above is plotted in Fig. 9 for $c_{L}=0.4$ with three different values of $c_{R}: 0.4,0.1,-0.2$. In the figure, we have plotted the mass eigenvalues of the top KK tower in units of $k^{\prime}$, obtained by solving Eq. (23) for given values of $c_{L}$ and $c_{R}$, as a function of $\lambda$. The solid, dashed and dot-dashed lines represent the spectra for $c_{R}=0.4,0.1$ and -0.2 , respectively. We can see from the figure that the masses approach to constant values for $\lambda \rightarrow \infty$. For the lowest mass eigenstate, which we identify as the standard-model top quark, the mass approaches to $m / k^{\prime} \simeq 0.65$ for $\lambda \rightarrow \infty$ regardless of the value of $c_{R}$, although the value of $m / k^{\prime}$ obtained for $\lambda=O(1)$ depends on $c_{R}$. This is because for $\lambda \rightarrow \infty$ the mass eigenvalues are determined by the condition that the second term in the left-hand-side of Eq. (23) is vanishing; 


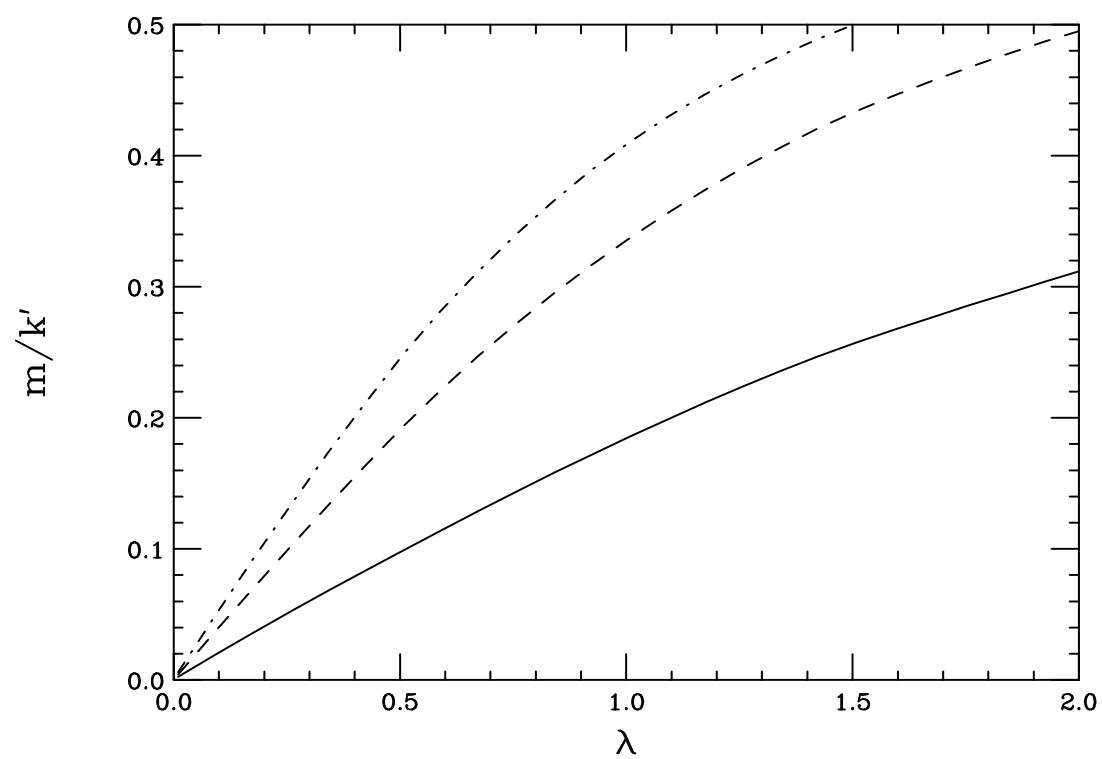

Figure 10: The same as Fig. 10 but for $0 \leq \lambda \leq 2$ and $0 \leq m / k^{\prime} \leq 0.5$.

namely, the sum of the solutions of $J_{c+1 / 2}\left(m / k^{\prime}\right)-\left(J_{c-1 / 2}(m / k) / Y_{c-1 / 2}(m / k)\right) Y_{c+1 / 2}\left(m / k^{\prime}\right)=0$ for $c=c_{L}$ and $c_{R}$. This in turn implies that the maximum value of the top-quark mass, i.e. the mass at $\lambda \rightarrow \infty$, is determined by the value of the largest of $c_{L}$ and $c_{R}\left(c_{L}\right.$ in the case of Fig. 9), since the value of $m / k^{\prime}$ obtained as the lowest mass solution of the above equation decreases for increasing value of $c$. Specifically, the maximum value of the top-quark mass is given by $m / k^{\prime} \simeq 0.25,0.45,0.65,0.80$ and 0.95 for $\max \left\{c_{L}, c_{R}\right\}=0.5,0.45,0.4,0.35$ and 0.3 , respectively. Therefore, there is a tension between the top-quark mass and the constraint from $Z \rightarrow b \bar{b}$ discussed at the end of the previous subsection, as $Z \rightarrow b \bar{b}$ provides the lower limit for the value of $c_{L}$ [22]. Since the value of $k^{\prime}$ in our theory is $k^{\prime} \simeq 2 \pi v / \sqrt{N}$ (from Eqs. (8) and (20)), we find that $N$ must satisfy $N \lesssim 4$ to obtain a large enough top-quark mass, $m_{t} \simeq 165 \mathrm{GeV}$ for $\overline{\mathrm{MS}}$ without QCD radiative corrections. ${ }^{10}$ We also find from Fig. 9 that for sufficiently large values for $c_{R}$ the mass of the first top-quark KK tower is significantly lighter than that of the first gauge-boson KK tower, $m_{1} \simeq 2.4 k^{\prime}$, in the region $\lambda \gg 1$. This feature, however, is lost for $\lambda \lesssim 1$ or smaller values of $c_{R}$.

In Fig. 10, we present a magnification of the lower-left corner of Fig. 9. From this figure we can obtain $\lambda$ for a given value of $N$. Let us, for example, consider the two cases of $N \simeq 1$ and 3 . In these cases, the values of $k^{\prime}$ are given by $k^{\prime} \simeq 1100 \mathrm{GeV}$ and $630 \mathrm{GeV}$, respectively, requiring

\footnotetext{
${ }^{10}$ Introducing Planck-brane localized kinetic terms for the left- and right-handed top quarks does not significantly modify the argument, unless there are unnatural cancellations.
} 
$m / k^{\prime} \simeq 0.15$ and 0.26 to reproduce the observed top-quark mass. These values are easily obtained for $c_{R}=\{-0.2,0.1,0.4\}$ by choosing $\lambda \simeq\{0.3,0.4,0.8\}$ and $\{0.5,0.7,1.5\}$, respectively. It is interesting that these values of $\lambda$ are what one would naively expect from dimensional grounds: $y_{u} \approx 1 / M_{*}$ and $H \approx M_{*}$. This is also true for larger values of $c_{L}$ and for the case of strong bulk gauge couplings. For example, for $c_{L}=0.49$ and $N \simeq 1$ one only needs $\lambda \simeq\{0.7,0.9,1.9\}$ for $c_{R}=\{-0.2,0.1,0.4\}$ to obtain the observed top-quark mass.

We finally discuss phenomenological issues in the top sector. As the $W$ and $Z$ wavefunctions are not flat in the extra dimension, the couplings of these gauge bosons to the top quark deviate from their standard model values. For the left-handed top quark, the deviation of the coupling to $Z$ is roughly given by Eq. (26) and at a level of a few percent. The deviation of the $t_{L} b_{L} W$ coupling from the standard model is also at this level. Therefore, these effects are not constrained by the present experimental data. The effects on the right-handed top quark can be much larger because it has smaller values of $c$. For example, smaller values of $c$ induce the interaction of the right-handed top quark $t_{R}$ to the $W$ boson. However, this interaction couples $t_{R}$ and $W$ only with the heavy field $\bar{B}$ and its KK tower, denoted as $\bar{D}$ in Eq. (22), so it is irrelevant at low energies. A particularly interesting effect appears in the right-handed top quark coupling to the $Z$ boson, $t_{R} t_{R} Z$. This coupling can have an order-one deviation from the standard model value. The deviation arises mainly from the fact that the $Z$ wavefunction inside $A_{\mu}^{R 3}$ has a non-trivial profile, and is approximately given by $\delta g_{R}^{t} / g_{R}^{t}=f^{\prime \prime} g_{R}^{2} /\left(g_{L}^{2}+g_{R}^{2}\right)$, where $f^{\prime \prime} \approx$ $\left(1-2 c_{R}\right) /\left(1-\left(k^{\prime} / k\right)^{1-2 c_{R}}\right)$ with $c_{R}$ representing the bulk mass for $t_{R}$. This is in contrast to the

case of the $b_{L} b_{L} Z$ coupling, which arises from the variation of the $Z$ wavefunction in $A_{\mu}^{L 3}$ and thus is given by $\delta g_{L}^{b} / g_{L}^{b}=f^{\prime} g_{L}^{2} /\left(g_{L}^{2}+g_{R}^{2}\right)$ with $f^{\prime} \approx\left(1-2 c_{L}\right) /\left(1-\left(k^{\prime} / k\right)^{1-2 c_{L}}\right)$ (see Eqs. $\left.(26,27)\right)$. Since $g_{R}$ can generically be larger then $g_{L}$ (and $c_{R}$ smaller than $c_{L}$ ), $\delta g_{R}^{t} / g_{R}^{t}$ can be large. It will be interesting to explore this coupling in a future $e^{+} e^{-}$linear collider [29].

\subsection{Corrections to oblique parameters}

From the analysis in section 4 of the electroweak oblique corrections generated by the gauge sector, we have found that there are two possibilities for making the theory viable. One is to have $N \simeq 1$, i.e. $\left(g_{L}^{2}+g_{R}^{2}\right) k \simeq 16 \pi^{2}$, and the other is to extend the theory to give an additional negative contribution to $S$. In the former case the contributions to $S$ and $T$ from the top sector cannot be reliably calculated, as the IR cutoff of the theory is close to the scale of the first resonance so that there is no energy region where the theory has a weak coupling description. However, an argument similar to the gauge case suggests that these contributions are of order $S \approx T \approx 1 / \pi$. The relevant diagrams are similar to those in Fig. 5 but with appropriate modifications (internal lines should be the top quark or its $S U(2)_{R}$ partner, there must be more insertions of the $G$ 
dynamics with the chirality flipping effects, and so on). Because the diagrams have insertions of the fermion masses, the contributions from the other fermions are much smaller. Therefore, although we do not know the precise contribution from the matter sector, we can expect that the theory is still viable in the sense that the contributions to the oblique parameters are of order $S, T \lesssim 1 / \pi$.

In the case with additional negative $S$ contributions, we can have a moderate energy interval where the theory is weakly coupled. In this case, the top contribution to $S$ does not give any additional constraint, because we have already invoked the cancellation between the gauge and the additional negative contributions - we simply have to make the sum of the gauge, top and additional contributions to be smaller than the experimental constraint. As for the top contribution to $T$, it is a calculable quantity dominated by the IR region $\approx k^{\prime}$, due to the custodial $S U(2)$ symmetry of the $G$ sector [22]. The contribution is roughly given by $T \approx a_{t}\left(m_{t} / k^{\prime}\right)^{2}$, where $a_{t}$ is a constant; the value of $a_{t}$ depends on $c_{L}$ and $c_{R}$, and may have an enhancement coming from the fact that the $G$ sector (KK towers) feels stronger chiral symmetry breaking than the elementary sector. The acceptable values of $T$ from the top sector depend on the size of the contributions from the gauge sector and an additional sector needed to make $S$ sufficiently small. However, based on the naive estimate, it does not seem implausible to expect that the top contribution, together with all the other contributions, actually fit to the data in some explicit models.

\subsection{Implications for flavor physics}

As mentioned in section 5.2, flavor violation in this model arises as a consequence of the need to have different bulk masses for the third generation quarks in order to obtain a large top quark mass. This non-universality leads to tree-level FCNCs. These have two main manifestations. First, since the wavefunction of the $Z$ is pushed away from the IR brane by the boundary conditions (or a large VEV), there will be non-negligible tree-level FCNC couplings of $q^{T}=$ $\left(t_{L} b_{L}\right)^{T}$ and $t_{R}$ with the $Z$, since these must be localized not too far from this brane. We define the effective $Z b s$ coupling by

$$
\mathcal{L}_{Z b s}=\frac{g^{2}}{4 \pi^{2}} \frac{g}{2 \cos \theta_{W}}\left(Z_{b s} \bar{b}_{L} \gamma^{\mu} s_{L}+Z_{b s}^{\prime} \bar{b}_{R} \gamma^{\mu} s_{R}\right) Z_{\mu}
$$

where $Z_{b s}$ and $Z_{b s}^{\prime}$ encode both the one-loop standard model as well as new physics contributions. Up to a factor of order one, the tree-level FCNC vertex induced by the flavor violating coupling discussed in section 5.2 results in

$$
\delta Z_{b s} \simeq\left(-\frac{1}{2}+\frac{1}{3} \sin ^{2} \theta_{W}\right) D_{L}^{b s} \frac{8 \pi^{2}}{g^{2}}\left(\frac{\delta g_{L}^{b}}{g_{L}^{b}}\right) \simeq-8 \pi^{2} D_{L}^{b s} \frac{\delta g_{L}^{b}}{g_{L}^{b}}
$$


where $\delta g_{L}^{b} / g_{L}^{b}$ is given in Eq. (26) and takes a value of order one percent (or somewhat smaller) in a generic parameter region. In Eq. (29), $D_{L}^{b s}$ corresponds to the $b$ - $s$ element of the lefthanded down-quark rotation diagonalizing the quark masses. Thus, with the natural assumption $D_{L}^{b s} \simeq V_{t b}^{*} V_{t s}$, the correction is of the same order as the standard model contribution to this vertex, which is $[30] Z_{b s}^{\mathrm{SM}} \simeq-0.04\left(Z_{b s}^{\mathrm{SM}} \simeq 0\right)$. This leads to potentially observable effects in $b \rightarrow s \ell^{+} \ell^{-}$ decays, although the current experimental data, $\left|Z_{b s}\right| \lesssim 0.08$ [30], is not greatly constraining. ${ }^{11}$ The effects are larger in the case that the bound on $Z \rightarrow b \bar{b}$ is saturated, i.e. $\delta g_{L}^{b} / g_{L}^{b} \simeq 1 \%$, for example in the case of strong bulk gauge couplings. The effect of Eq. (29) also contributes to hadronic modes, such as $B \rightarrow \phi K_{s}$, although there it must compete with the parametrically larger contributions from gluonic penguins.

The second type of tree-level FCNC effects occur in the interactions of third generation quarks with the all KK gauge bosons, including those that belong to an unbroken symmetry, such as the KK gluons. This is due to the fact that the lightest KK gauge bosons are localized toward the IR brane, and are therefore strongly coupled to $t_{L}, b_{L}$ and $t_{R}$. The FCNC interactions of KK gluons with $b_{L}$ also lead to contributions to hadronic $B$ decays, and they are potentially of the same order or even larger than the standard model gluonic penguins. These could result in sizeable deviations in $\mathrm{CP}$ asymmetries in $B$ decays such as $B \rightarrow \phi K_{s}, B \rightarrow \eta^{\prime} K_{s}$ and $B \rightarrow \pi^{0} K_{s}$, among others [32], even after the constrains from $Z \rightarrow b \bar{b}$ are taken into account.

Finally, the large flavor violating coupling of the top quark, particularly $t_{R}$, may lead to a large contribution to $D^{0}-\bar{D}^{0}$ mixing. This has the contributions both from $\mathrm{KK}$ gluon and $Z$ exchanges and has the form

$$
\Delta m_{D} \simeq 4 \pi \alpha_{s} \frac{\chi\left(c_{R}\right)}{2 m_{1}^{2}} \frac{\left(U_{R}^{t u *} U_{R}^{t c}\right)^{2}}{2 m_{D}}\left\langle D^{0}\left|\left(\bar{c}_{R} \gamma_{\mu} u_{R}\right)\left(\bar{c}_{R} \gamma^{\mu} u_{R}\right)\right| \bar{D}^{0}\right\rangle,
$$

for the KK gluon exchange. Here, $U_{R}$ is the rotation matrix for right-handed up quarks, and $\chi\left(c_{R}\right)$ is a function of $c_{R}$ which gives the enhancement due to the strong coupling of the KK gluons to $t_{R}$. For instance, for $c_{R} \simeq 0$ and small brane couplings, $\chi \simeq 16$. To estimate the contribution to $\Delta m_{D}$, we need the quark rotation matrix elements. If we take $U_{R}^{t u *} U_{R}^{t c} \simeq \sin ^{5} \theta_{C}$, with $\sin \theta_{C} \simeq 0.2$ the Cabibbo angle, then the current experimental limit [33] on $\Delta m_{D}$ translates into $m_{1} \gtrsim 2 \mathrm{TeV} .{ }^{12}$ In the strong bulk coupling case, $\chi\left(c_{R}\right)$ can be enhanced and somewhat larger $c_{R}$ or smaller mixing angles may be required. The contribution from $Z$ is generically the same order but somewhat smaller. We thus find that the effect can be consistent with but naturally close to the current experimental limit. Similar contributions come from the interactions of $t_{L}$, but they are typically smaller than those from $t_{R}$ because of larger values of $c$.

\footnotetext{
${ }^{11}$ This possibility has also been mentioned in [31] in the context of the model of [22]. We thank K. Agashe for pointing out this reference to us.

${ }^{12}$ Unlike for $U_{L}$ and $D_{L}$, there is in principle no reason why $U_{R}$ must have such scaling with the Cabibbo angle.
} 


\section{Conclusions and Discussion}

We have studied theories of electroweak symmetry breaking without a Higgs boson. The electroweak symmetry is broken by non-trivial dynamics of a gauge interaction $G$, whose gauge coupling and the number of "colors" are denoted as $\tilde{g}$ and $N$, respectively. In conventional 4D technicolor, the theory is assumed to be weakly coupled at the UV - the loop expansion parameter $\kappa \equiv \tilde{g}^{2} N / 16 \pi^{2}$ is smaller than unity — and electroweak symmetry breaking is triggered at the IR where the perturbative expansion breaks down, $\kappa \simeq 1$. This makes the theory intractable because we must sum up all contributions of the form $\kappa^{n}(n \in \mathbf{Z})$ arising at the $n$-th order in perturbation theory, to compute quantities such as the electroweak oblique parameters $S$ and T. Moreover, such a theory generically has the problem of generating realistic fermion masses without conflicting with the experimental constraints on flavor violation.

In this paper we have studied an alternative possibility of "dynamical" electroweak symmetry breaking, in which the parameter $\kappa$ is larger than unity at the UV. In particular, we have concentrated on the case where $\kappa$ stays almost constant over a wide energy range above the electroweak scale, as indicated by the curve (b) in Fig. 1. In such a theory quantities such as $S$ and $T$ can in principle be calculable because they are given as expansions in powers of $1 / \kappa$ and $1 / N$. With sufficiently large $\kappa$ and $N$, therefore, we expect to have a calculable theory of electroweak symmetry breaking. This is actually the case for a certain theory of this type, where there is a dual description in terms of a 5D theory compactified on the truncated AdS space. In this dual description, the electroweak symmetry is simply broken by boundary conditions imposed at a spacetime boundary (the IR brane). As long as the theory is weakly coupled and the AdS curvature scale $k$ is sufficiently small - which correspond in the 4D picture to have sufficiently large $\kappa$ and $N$ - we can reliably compute $S$ and $T$. Quark and lepton masses are also obtained relatively easily by putting these fields in the 5D bulk and giving them masses at the IR brane.

Because the theory is calculable, it is possible to make a reliable comparison between its predictions and experimental data. We have explicitly computed the gauge contribution to the $S$ parameter in the simplest potentially realistic theory of the kind discussed above - a 5D warped space theory with the electroweak symmetry broken by the boundary conditions at the IR brane, and with the custodial $S U(2)$ symmetry imposed on the $G$ sector encoded in the physics of the $5 \mathrm{D}$ bulk and the IR boundary. The result can be written in the form $S=c_{S} N / \pi$ (or $S=c_{S} N^{2} / \pi$ ), where $c_{S}$ is a positive constant of order one and $N$ (or $N^{2}$ ) is given by $16 \pi^{2} /\left(g_{L}^{2}+g_{R}^{2}\right) k$ with $g_{L}$ and $g_{R}$ representing the 5D gauge couplings of the bulk gauge groups $S U(2)_{L}$ and $S U(2)_{R}$, respectively. This result has a striking similarity to the estimate of $S$

in technicolor theories. This is because the size of the prediction of the theory for a physical 
quantity $P$ is in general rather insensitive to the value of $\kappa$ - expanding $P$ in powers of $1 / N$ as $P=\sum_{n} f_{n}(\kappa) N^{-n}$, the dependence of the functions $f_{n}(\kappa)$ on $\kappa$ is rather mild: $f_{n}(\kappa)=O(1)$ for the entire range of $\kappa$. This in turn implies that if we want to have a large value of $N$, or equivalently a large energy interval where the theory has a weakly coupled 5D gravitational description, the gauge contribution to $S$ must be canceled by some other negative contribution, which does not arise from the gauge or matter sector of the model. While such a contribution may arise, perhaps, from the intrinsic structure of the theory, for example from the sector needed to stabilize the radius of the extra dimension, it will most likely require an extension of the model. The amount of cancellation required is typically of order $10 \%$, but if it is attained, we can have a weakly coupled, calculable theory of "dynamical" electroweak symmetry breaking.

Another interesting possibility is to have a value of $N$ close to unity, which is attained by making one or both of the $5 \mathrm{D}$ gauge couplings $g_{L}$ and $g_{R}$ large. In this case we loose the calculability of the theory, but we expect that the corrections to the electroweak oblique parameters are small and of order $1 / \pi$. Realistic quark and lepton masses will also be obtained without contradicting with bounds from flavor changing processes, as this property is expected to persist even as we make the 5D theory strongly coupled. We therefore arrive at a potentially consistent picture of a dynamical theory of electroweak symmetry breaking - our theory is obtained by taking the limit of strong 5D gauge couplings in a warped $5 \mathrm{D}$ theory, in which the electroweak symmetry is broken by boundary conditions imposed at the IR brane.

A particularly interesting version of this theory is obtained by taking all the $5 \mathrm{D}$ gauge couplings to be strong:

$$
M_{*} \simeq \pi k \simeq \frac{16 \pi^{3}}{g_{5 \mathrm{D}}^{2}},
$$

where $M_{*}$ is the cutoff scale of the theory and $g_{5 \mathrm{D}}$ represents all the 5D bulk gauge couplings $g_{C}, g_{L}, g_{R}$ and $g_{X}$ for $S U(3)_{C}, S U(2)_{L}, S U(2)_{R}$ and $U(1)_{X}$. The observed gauge couplings then come almost entirely from the Planck-brane couplings. The oblique parameters are expected to have a size

$$
S, T \lesssim \frac{1}{\pi}
$$

which is reasonably small, given the uncertainty of the estimate. Because $M_{*} \simeq \pi k$ implies that the IR cutoff scale $M_{*}^{\prime}$ is close to the mass of the first $\mathrm{KK}$ resonance $m_{1} \simeq \pi k^{\prime}$, where $M_{*}^{\prime} \equiv M_{*} e^{-\pi k R}$ and $k^{\prime} \equiv k e^{-\pi k R}$, we do not have a weakly coupled KK picture around the TeV scale. Rather, we have strongly coupled "string states" at the scale of

$$
M_{*}^{\prime} \simeq 4 \pi v \simeq 2 \mathrm{TeV}
$$

below which the theory is essentially the standard model without a Higgs boson (the radion field may also be lighter than $M_{*}^{\prime}$ ). A difference from the conventional 4D technicolor picture 
is that above this scale these states become associated with the $4 \mathrm{D}$ gauge interaction $G$ but whose gauge coupling $\tilde{g}$ stays almost constant and has a value of order $4 \pi$ or slightly larger, i.e. $\kappa \simeq 1$ or slightly larger $\left(\kappa\right.$ in $4 \mathrm{D}$ corresponds to $M_{*} /(\pi k)$ in $\left.5 \mathrm{D}\right)$. Just above $M_{*}^{\prime}$, there are resonances, which then become increasingly broader and finally merge into a continuum consistent with conformal symmetry. This makes it possible to consider the theory as a strong coupling limit of a 5D warped theory. We can then expect that realistic fermion masses are obtained without phenomenological disasters, although some flavor violating signals could be close to the experimental bounds. This picture is also different from that of [34], because the theory as formulated in $4 \mathrm{D}$ is well defined up to the scale close to the $4 \mathrm{D}$ Planck scale, $M_{\mathrm{Pl}}$. The theory in the energy interval between $M_{*}^{\prime}$ and $M_{\mathrm{Pl}}$ is simply $S U(3)_{C} \times S U(2)_{L} \times U(1)_{Y} \times G$ gauge theory with the coupling of $G$ nearly constant and of order $4 \pi$. For example, the running of the $S U(3)_{C}, S U(2)_{L}$ and $U(1)_{Y}$ gauge couplings is still logarithmic with the beta-function coefficients given by

$$
b_{i}=b_{i}^{\mathrm{SM}}+\epsilon_{i},
$$

where $i=3,2,1$ represents $S U(3)_{C}, S U(2)_{L}$ and $U(1)_{Y}$, and $b_{i}^{\mathrm{SM}}$ are the standard-model beta functions without a Higgs boson, $\left(b_{1}, b_{2}, b_{3}\right)=(4,-10 / 3,-7)$ [in the " $S U(5)$ normalization" for the $U(1)_{Y}$ gauge coupling]; $\epsilon_{i}$ are the corrections arising from the $G$ sector and $\epsilon_{i} \simeq 1$. This may even suggest some sort of gauge unification at a high scale of order the Planck scale, since $\epsilon_{i}$ do not have an enhancement from the group theoretical factor so that the gross feature of the standard-model gauge coupling evolution (the three gauge couplings approach at high energies) is expected to persist. The entire physical picture of our theory is depicted in Fig. 11.

The strong coupling feature of the theory raises the issue that the 5D gravitational description may not be entirely trustable. For example, large quantum gravitational corrections may destabilize the structure of the background geometry. However, we only need essentially the AdS-like structure to be preserved in the 5D bulk so that there remains a large energy interval above the electroweak scale where the theory is near conformal. This may be the case, for example, when the 5D Planck scale $M_{5}$ is parametrically larger than the cutoff scale $M_{*}$. The full treatment of the theory will probably require string theoretic constructions, which may also give additional constraints: for example, the value of $N$ may be quantized through the Dirac quantization condition for higher-form gauge fields. Nevertheless, given the presence of an effective field theoretic model with a certain strong coupling limit, it does not seem so implausible to expect that the theory as described here does in fact exist in some UV-completed schemes.

The experimental signatures of such a theory will be quite "simple". Below the scale of $\simeq 2 \mathrm{TeV}$, the theory is essentially the standard model without a Higgs boson. New states appear at $M_{*}^{\prime} \simeq 2 \mathrm{TeV}$, which are composite states of the $G$ sector and will effectively be described as "string" states. Since some of these states will be unstable, the tail of this physics may show up 


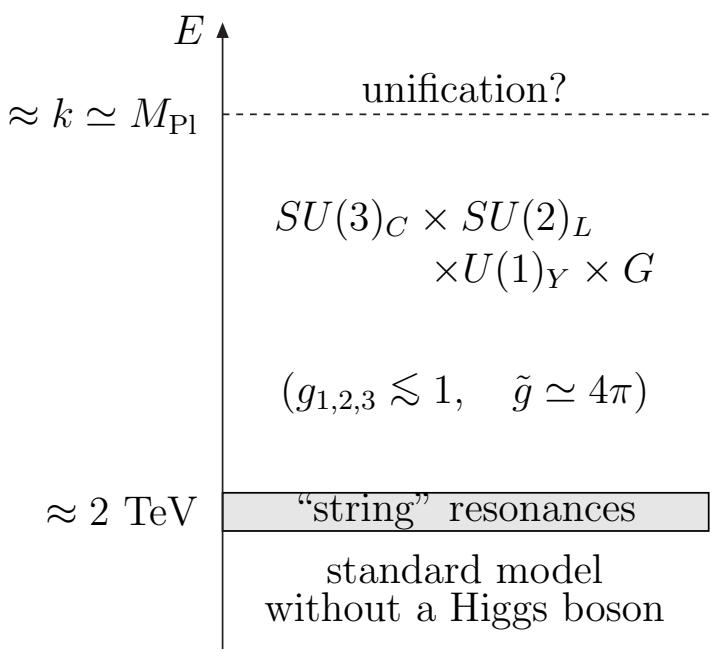

Figure 11: The overall picture of the theory. Here $g_{1,2,3}$ represents the gauge couplings of $S U(3)_{C} \times S U(2)_{L} \times U(1)_{Y}$, while $\tilde{g}$ represents that of $G$.

even at lower energies in collider experiments. This situation is similar to the minimal technicolor theory, but here the observed fermion masses are correctly reproduced through physics at higher energies. In this respect, it may not be easy to discriminate the present theory from certain technicolor models [35] (they may even be related to each other in the space of $\kappa$ ). An interesting state is the radion field which is expected to be lighter than $M_{*}^{\prime}$, especially when $M_{5} \gtrsim M_{*}$, and which arises in the 4D picture by spontaneous breaking of conformal invariance. The properties of this field are similar to the standard model Higgs boson in some parameter region, but in general can be different [36]. Because the theory can tell quite little about physics at the $2 \mathrm{TeV}$ scale, it will be very important to explore this energy region experimentally. Through such explorations, we will be able to learn about the physics of electroweak symmetry breaking caused by strong dynamics that does not contain any small parameter.

\section{Note added:}

After the completion of this work, Ref. [37] appeared which addresses related issues.

\section{Acknowledgments}

This work was supported in part by the Director, Office of Science, Office of High Energy and Nuclear Physics of the U.S. Department of Energy under Contract DE-AC0376SF00098. 


\section{Appendix A}

In this appendix we calculate the oblique parameters $S, T$ and $U$ at the leading order for the theory described in section 3 . These parameters can be calculated by integrating out the new physics, i.e. the $G$ sector, and deriving the low-energy effective theory for the electroweak gauge bosons. The effects of the $G$ sector then appear in the vacuum polarizations for these gauge fields, which we parameterize by $\Pi_{X Y}$ and $\Pi_{X Y}^{\prime}(X, Y=1,3, Q)$ as

$$
\begin{aligned}
\mathcal{L}_{\mathrm{eff}}= & -\frac{1}{2} \sum_{a=1}^{2} W_{\mu}^{a}\left[\left(\frac{v^{2}}{2}+\Pi_{11}\right)+p^{2}\left(\frac{1}{g^{2}}-\Pi_{11}^{\prime}\right)\right] W_{\mu}^{a} \\
& -\frac{1}{2} W_{\mu}^{3}\left[\left(\frac{v^{2}}{2}+\Pi_{33}\right)+p^{2}\left(\frac{1}{g^{2}}-\Pi_{33}^{\prime}\right)\right] W_{\mu}^{3} \\
& -\frac{1}{2} B_{\mu}\left[\left(\frac{v^{2}}{2}+\Pi_{33}\right)+p^{2}\left(\frac{1}{g^{\prime 2}}-\Pi_{33}^{\prime}+2 \Pi_{3 Q}^{\prime}-\Pi_{Q Q}^{\prime}\right)\right] B_{\mu} \\
& +W_{\mu}^{3}\left[\left(\frac{v^{2}}{2}+\Pi_{33}\right)-p^{2}\left(\Pi_{33}^{\prime}-\Pi_{3 Q}^{\prime}\right)\right] B_{\mu},
\end{aligned}
$$

where $\mathcal{L}_{\text {eff }}$ is the low-energy effective Lagrangian in the $4 \mathrm{D}$ momentum space. The normalizations for the gauge fields are taken such that they couple to the matter fields through the covariant derivatives

$$
\begin{aligned}
\mathcal{D}_{\mu} \psi_{L} & =\partial_{\mu} \psi_{L}+\frac{i}{2}\left(\begin{array}{cc}
W_{\mu}^{3}+2 Y B_{\mu} & W_{\mu}^{1}-i W_{\mu}^{2} \\
W_{\mu}^{1}+i W_{\mu}^{2} & -W_{\mu}^{3}+2 Y B_{\mu}
\end{array}\right) \psi_{L}, \\
\mathcal{D}_{\mu} \psi_{R} & =\partial_{\mu} \psi_{R}+i Y B_{\mu} \psi_{R},
\end{aligned}
$$

where $\psi_{L}$ and $\psi_{R}$ represent $\{q, l\}$ and $\{u, d, e\}$, respectively. The parameters $S, T$ and $U$ are defined by [9]

$$
\begin{aligned}
S & \equiv 16 \pi\left(\Pi_{33}^{\prime}-\Pi_{3 Q}^{\prime}\right) \\
T & \equiv \frac{8 \pi\left(g^{2}+g^{\prime 2}\right)}{g^{2} g^{\prime 2} v^{2}}\left(\Pi_{11}-\Pi_{33}\right) \\
U & \equiv 16 \pi\left(\Pi_{11}^{\prime}-\Pi_{33}^{\prime}\right)
\end{aligned}
$$

These parameters do not depend on the values of $g$ and $g^{\prime}$ we choose to extract $\Pi s$ and $\Pi^{\prime}$ s from Eq. (35), as long as $\Pi_{11,33} \ll v^{2}$ and $\Pi_{11,33,3 Q, Q Q}^{\prime} \ll 1 / g^{2}, 1 / g^{\prime 2}$.

The effective Lagrangian, $\mathcal{L}_{\text {eff }}$, in our theory is obtained in $5 \mathrm{D}$ by integrating out the physics of $y>0$ keeping the values of the $5 \mathrm{D}$ gauge fields at $y=0$ fixed, which we identify as the low-energy 4D fields [8]. Since we are here interested in the leading-order contributions to $S, T$ and $U$, which are represented in the $4 \mathrm{D}$ picture by the diagram shown in Fig. 2 in section 4, it 
is sufficient to solve the equations of motion for the bulk gauge fields at the classical level under appropriate boundary conditions.

The action for the gauge fields are given by Eqs. $(5,18)$, i.e. the sum of the bulk, Planckbrane, and TeV-brane gauge kinetic terms. In terms of the conformal coordinate $z \equiv e^{k y} / k$, the bulk equations of motion for the gauge fields are written as

$$
z \partial_{z}\left(\frac{1}{z} \partial_{z} A_{\mu}^{G}\right)-p^{2} A_{\mu}^{G}=0
$$

where we have kept only the transverse modes, and $G$ runs for $L 1, R 1, L 3, R 3$, and $X$, which represent the first two components of $S U(2)_{L}$, those of $S U(2)_{R}$, the third component of $S U(2)_{L}$, that of $S U(2)_{R}$, and $U(1)_{X}$, respectively. These equations have solutions of the form

$$
A_{\mu}^{G}(p, z)=z\left\{a_{\mu}^{G}(p) I_{1}(p z)+b_{\mu}^{G}(p) K_{1}(p z)\right\}
$$

where $I_{1}(x)$ and $K_{1}(x)$ are the modified Bessel functions, and $a_{\mu}^{G}(p)$ and $b_{\mu}^{G}(p)$ are functions of the $4 \mathrm{D}$ momentum $p$. The boundary conditions at the Planck brane $(y=0)$ are given by

$$
\begin{aligned}
{\left[A_{\mu}^{L 1}(p, z)\right]_{z=\frac{1}{k}} } & =W_{\mu}^{1}(p), \\
{\left[A_{\mu}^{R 1}(p, z)\right]_{z=\frac{1}{k}} } & =0, \\
{\left[A_{\mu}^{L 3}(p, z)\right]_{z=\frac{1}{k}} } & =W_{\mu}^{3}(p), \\
{\left[A_{\mu}^{R 3}(p, z)\right]_{z=\frac{1}{k}} } & =B_{\mu}(p), \\
{\left[A_{\mu}^{X}(p, z)\right]_{z=\frac{1}{k}} } & =B_{\mu}(p),
\end{aligned}
$$

which identify the 5D fields at the Planck brane to the low-energy 4D degrees of freedom. The boundary conditions at the TeV brane $(y=\pi R)$ are given by

$$
\begin{aligned}
& {\left[A_{\mu}^{L 1}(p, z)-A_{\mu}^{R 1}(p, z)\right]_{z=\frac{1}{k^{\prime}}}=0,} \\
& {\left[\frac{1}{g_{L}^{2}} \partial_{z} A_{\mu}^{L 1}(p, z)+\frac{1}{g_{R}^{2}} \partial_{z} A_{\mu}^{R 1}(p, z)+p^{2} \frac{k}{k^{\prime}}\left(\tilde{Z}_{L} A_{\mu}^{L 1}(p, z)+\tilde{Z}_{R} A_{\mu}^{R 1}(p, z)\right)\right]_{z=\frac{1}{k^{\prime}}}=0,} \\
& {\left[A_{\mu}^{L 3}(p, z)-A_{\mu}^{R 3}(p, z)\right]_{z=\frac{1}{k^{\prime}}}=0,} \\
& {\left[\frac{1}{g_{L}^{2}} \partial_{z} A_{\mu}^{L 3}(p, z)+\frac{1}{g_{R}^{2}} \partial_{z} A_{\mu}^{R 3}(p, z)+p^{2} \frac{k}{k^{\prime}}\left(\tilde{Z}_{L} A_{\mu}^{L 3}(p, z)+\tilde{Z}_{R} A_{\mu}^{R 3}(p, z)\right)\right]_{z=\frac{1}{k^{\prime}}}=0,} \\
& {\left[\frac{1}{g_{X}^{2}} \partial_{z} A_{\mu}^{X}(p, z)+p^{2} \frac{k}{k^{\prime}} Z_{X} A_{\mu}^{X}(p, z)\right]_{z=\frac{1}{k^{\prime}}}=0,}
\end{aligned}
$$

which are essentially those of Eq. (3) but appropriately modified by the presence of the TeVbrane gauge kinetic terms. Here, $\tilde{Z}_{L} \equiv Z_{L}+Z_{M}$ and $\tilde{Z}_{R} \equiv Z_{R}+Z_{M}$. 
The boundary conditions Eqs. $(43-52)$ determine the coefficients $a_{\mu}^{G}(p)$ and $b_{\mu}^{G}(p)$ in Eq. (42). Plugging these solutions into the original action, Eq. (5) and Eq. (18), and integrating over $z$, we obtain the low-energy effective Lagrangian. Expanding this Lagrangian in powers of $p$ up to the quadratic order, we find

$$
\begin{aligned}
& \mathcal{L}_{\text {eff }}=- \frac{k^{\prime 2}}{\left(g_{L}^{2}+g_{R}^{2}\right) k}\left(\sum_{a=1}^{2} W_{\mu}^{a} W_{\mu}^{a}+W_{\mu}^{3} W_{\mu}^{3}-2 W_{\mu}^{3} B_{\mu}+B_{\mu} B_{\mu}\right) \\
&-\frac{p^{2}}{2}\left\{\left(\frac{\pi R}{g_{L}^{2}}+\frac{1}{\tilde{g}_{L}^{2}}\right)\left(\sum_{a=1}^{2} W_{\mu}^{a} W_{\mu}^{a}+W_{\mu}^{3} W_{\mu}^{3}\right)+\left(\frac{\pi R}{g_{R}^{2}}+\frac{\pi R}{g_{X}^{2}}+\frac{1}{\tilde{g}_{Y}^{2}}\right) B_{\mu} B_{\mu}\right\} \\
&-\frac{p^{2}}{8\left(g_{L}^{2}+g_{R}^{2}\right) k}\left\{\left(-3+\frac{4 g_{R}^{4} k}{g_{L}^{2}+g_{R}^{2}}\left(\tilde{Z}_{L}+\tilde{Z}_{R}\right)\right)\left(\sum_{a=1}^{2} W_{\mu}^{a} W_{\mu}^{a}+W_{\mu}^{3} W_{\mu}^{3}\right)\right. \\
&+\left(-3+\frac{4 g_{L}^{4} k}{g_{L}^{2}+g_{R}^{2}}\left(\tilde{Z}_{L}+\tilde{Z}_{R}\right)+4\left(g_{L}^{2}+g_{R}^{2}\right) k Z_{X}\right) B_{\mu} B_{\mu} \\
&+\left.\left(6+\frac{8 g_{L}^{2} g_{R}^{2} k}{g_{L}^{2}+g_{R}^{2}}\left(\tilde{Z}_{L}+\tilde{Z}_{R}\right)\right) W_{\mu}^{3} B_{\mu}\right\} .
\end{aligned}
$$

This reproduces the matching relations of Eqs. (8) at the leading order in $1 / \pi k R$ (assuming $Z_{L, R, M, X}=O\left(1 / 16 \pi^{2}\right)$ as suggested by NDA). We then obtain the vacuum polarization parameters

$$
\begin{aligned}
& \Pi_{11}=\Pi_{33}=0, \\
& \Pi_{11}^{\prime}=\Pi_{33}^{\prime}=\frac{3}{4\left(g_{L}^{2}+g_{R}^{2}\right) k}-\frac{g_{R}^{4}}{\left(g_{L}^{2}+g_{R}^{2}\right)^{2}}\left(\tilde{Z}_{L}+\tilde{Z}_{R}\right), \\
& \Pi_{3 Q}^{\prime}=-\frac{g_{R}^{2}}{g_{L}^{2}+g_{R}^{2}}\left(\tilde{Z}_{L}+\tilde{Z}_{R}\right), \\
& \Pi_{Q Q}^{\prime}=-\left(\tilde{Z}_{L}+\tilde{Z}_{R}+Z_{X}\right) .
\end{aligned}
$$

Several features of this result can be understood from the symmetry reason. For example, the reason for why $\Pi_{11}=\Pi_{33}$ and $\Pi_{11}^{\prime}=\Pi_{33}^{\prime}$ comes from the fact that the dynamics of the $G$ sector, encoded in the bulk and TeV-brane physics, respects the custodial $S U(2)$ symmetry. The coefficients for TeV-brane operators $Z_{L}, Z_{R}$ and $Z_{M}$ always appear in the combination of $\tilde{Z}_{L}+\tilde{Z}_{R}=Z_{L}+Z_{R}+Z_{M}$ because the $\mathrm{TeV}$ brane respects the diagonal subgroup of $S U(2)_{L}$ and $S U(2)_{R}$. Finally, in the case of vanishing TeV-brane operators, $Z_{L}=Z_{R}=Z_{M}=Z_{X}=0$, we get $\Pi_{3 Q}^{\prime}=\Pi_{Q Q}^{\prime}=0$. This is because the full $S U(2)_{L} \times S U(2)_{R}$ symmetry is respected by the operators in the $5 \mathrm{D}$ bulk.

Eqs. $(54-57)$ give the oblique parameters

$$
\begin{aligned}
S & =\frac{16 \pi}{\left(g_{L}^{2}+g_{R}^{2}\right) k}\left\{\frac{3}{4}+\frac{g_{L}^{2} k \cdot g_{R}^{2} k}{\left(g_{L}^{2}+g_{R}^{2}\right) k}\left(\tilde{Z}_{L}+\tilde{Z}_{R}\right)\right\}, \\
T & =U=0 .
\end{aligned}
$$


As expected, the $T$ and $U$ parameters are zero at this order because of the custodial $S U(2)$ symmetry imposed on the $G$ sector. The value of $S$ has a size of order $N / \pi$, where $N$ is given by Eq. (13), as discussed in section 4.1 .

\section{Appendix B}

In this appendix we derive the formula determining the mass eigenvalues for the quark and lepton KK towers. We here use the notation of Eq. (22) for the up-type quark sector. However, the computation is completely identical for the down-type quark and lepton sectors, so that the results are also applicable to these cases.

We define the rescaled fields $\hat{u}=e^{-2 k y} u, \hat{u}^{c}=e^{-2 k y} u^{c}, \hat{\bar{u}}=e^{-2 k y} \bar{u}$, and $\hat{\bar{u}}^{c}=e^{-2 k y} \bar{u}^{c}$. In terms of these fields, the action is written as

$$
\begin{aligned}
\mathcal{S}= & \int d^{4} x \int d y\left[e^{k y}\left(\hat{u}^{\dagger} i \bar{\sigma}^{\mu} \partial_{\mu} \hat{u}+\hat{u}^{c} i \sigma^{\mu} \partial_{\mu} \hat{u}^{c \dagger}+\hat{\bar{u}} i \sigma^{\mu} \partial_{\mu} \hat{\bar{u}}^{\dagger}+\hat{\bar{u}}^{c \dagger} i \bar{\sigma}^{\mu} \partial_{\mu} \hat{\bar{u}}^{c}\right)\right. \\
& +\hat{u}^{c}\left(\partial_{y}+c_{L} k\right) \hat{u}+\hat{u}^{\dagger}\left(-\partial_{y}+c_{L} k\right) \hat{u}^{c \dagger}+\hat{\bar{u}}^{c}\left(\partial_{y}+c_{R} k\right) \hat{\bar{u}}+\hat{\bar{u}}^{\dagger}\left(-\partial_{y}+c_{R} k\right) \hat{\bar{u}}^{c \dagger} \\
& \left.-\delta(y-\pi R)\left(\lambda \hat{u} \hat{\bar{u}}+\lambda^{*} \hat{u}^{\dagger} \hat{\bar{u}}^{\dagger}\right)\right],
\end{aligned}
$$

where the bulk terms come from the $5 \mathrm{D}$ kinetic terms and the TeV-brane terms from the operator in Eq. (7); $c_{L}$ and $c_{R}$ are the bulk mass parameters for the $q$ and $\psi_{\bar{u}}$ fields, i.e. $c_{L}=c_{q}$ and $c_{R}=c_{\psi_{\bar{u}}}$, and $\lambda$ is given by $\lambda=y_{u} v_{H}$.

The above action, Eq. (60), provides both bulk equations of motion and boundary conditions. Expanding the 5D fields as

$$
\begin{aligned}
\hat{u}(x, y) & =u(x) \hat{f}_{u}(y), \\
\hat{u}^{c}(x, y) & =\bar{u}(x) \hat{f}_{u}^{c}(y), \\
\hat{\bar{u}}(x, y) & =\bar{u}(x) \hat{f}_{\bar{u}}(y), \\
\hat{\bar{u}}^{c}(x, y) & =u(x) \hat{f}_{\bar{u}}^{c}(y),
\end{aligned}
$$

we obtain the bulk equations of motion

$$
\begin{aligned}
& \left(-\partial_{z}+\frac{c_{L}}{z}\right) f_{u}^{c}+m f_{u}=0, \\
& \left(\partial_{z}+\frac{c_{L}}{z}\right) f_{u}+m f_{u}^{c}=0, \\
& \left(-\partial_{z}+\frac{c_{R}}{z}\right) f_{\bar{u}}^{c}+m f_{\bar{u}}=0, \\
& \left(\partial_{z}+\frac{c_{R}}{z}\right) f_{\bar{u}}+m f_{\bar{u}}^{c}=0,
\end{aligned}
$$


where $m$ represents the $4 \mathrm{D}$ mass eigenvalues, $i \bar{\sigma}^{\mu} \partial_{\mu} q=m \bar{q}^{\dagger}$. Here, we have used the conformal coordinate $z \equiv e^{k y} / k$, and $f_{u}(z)=\hat{f}_{u}(\ln (k z) / k), f_{u}^{c}=\hat{f}_{u}^{c}(\ln (k z) / k), f_{\bar{u}}=\hat{f}_{\bar{u}}(\ln (k z) / k)$ and $f_{\bar{u}}^{c}=\hat{f}_{\bar{u}}^{c}(\ln (k z) / k)$. The boundary conditions are given by

$$
\left\{\begin{array} { l } 
{ f _ { u } ^ { c } | _ { z = \frac { 1 } { k } + \epsilon } = 0 , } \\
{ ( \partial _ { z } + k c _ { L } ) f _ { u } | _ { z = \frac { 1 } { k } + \epsilon } = 0 , } \\
{ f _ { \overline { u } } ^ { c } | _ { z = \frac { 1 } { k } + \epsilon } = 0 , } \\
{ ( \partial _ { z } + k c _ { R } ) f _ { \overline { u } } | _ { z = \frac { 1 } { k } + \epsilon } = 0 , }
\end{array} \quad \left\{\begin{array}{l}
\left.f_{u}^{c}\right|_{z=\frac{1}{k^{\prime}}-\epsilon}-\left.\lambda f_{\bar{u}}\right|_{z=\frac{1}{k^{\prime}}-\epsilon}=0 \\
\left.\left(\partial_{z}+k^{\prime} c_{L}\right) f_{u}\right|_{z=\frac{1}{k^{\prime}}-\epsilon}+\left.m f_{u}^{c}\right|_{z=\frac{1}{k^{\prime}}-\epsilon}=0 \\
\left.f_{\bar{u}}^{c}\right|_{z=\frac{1}{k^{\prime}}-\epsilon}-\left.\lambda f_{u}\right|_{z=\frac{1}{k^{\prime}}-\epsilon}=0, \\
\left.\left(\partial_{z}+k^{\prime} c_{R}\right) f_{\bar{u}}\right|_{z=\frac{1}{k^{\prime}}-\epsilon}+\left.m f_{\bar{u}}^{c}\right|_{z=\frac{1}{k^{\prime}}-\epsilon}=0
\end{array}\right.\right.
$$

where $\epsilon \rightarrow 0$. In Eqs. $(65-69)$, we have rotated the phases of the fields such that the coupling $\lambda$, and thus the 4D masses $m$, becomes real.

Eqs. $(65-68)$ have the solutions of the form

$$
\begin{aligned}
f_{u} & =\sqrt{z}\left\{a_{u} J_{c_{L}+\frac{1}{2}}(m z)+b_{u} Y_{c_{L}+\frac{1}{2}}(m z)\right\}, \\
f_{u}^{c} & =\sqrt{z}\left\{a_{u}^{c} J_{c_{L}-\frac{1}{2}}(m z)+b_{u}^{c} Y_{c_{L}-\frac{1}{2}}(m z)\right\}, \\
f_{\bar{u}} & =\sqrt{z}\left\{a_{\bar{u}} J_{c_{R}+\frac{1}{2}}(m z)+b_{\bar{u}} Y_{c_{R}+\frac{1}{2}}(m z)\right\}, \\
f_{\bar{u}}^{c} & =\sqrt{z}\left\{a_{\bar{u}}^{c} J_{c_{R}-\frac{1}{2}}(m z)+b_{\bar{u}}^{c} Y_{c_{R}-\frac{1}{2}}(m z)\right\},
\end{aligned}
$$

where $a_{u}, b_{u}, a_{u}^{c}, b_{u}^{c}, a_{\bar{u}}, b_{\bar{u}}, a_{\bar{u}}^{c}$ and $b_{\bar{u}}^{c}$ are constants. These constants are determined by the boundary conditions, Eq. (69). Non-trivial solutions are then obtained only when the following relation is satisfied:

$$
\begin{aligned}
& \left(J_{c_{L}-\frac{1}{2}}\left(\frac{m}{k^{\prime}}\right)-\frac{J_{c_{L}-\frac{1}{2}}\left(\frac{m}{k}\right)}{Y_{c_{L}-\frac{1}{2}}\left(\frac{m}{k}\right)} Y_{c_{L}-\frac{1}{2}}\left(\frac{m}{k^{\prime}}\right)\right)\left(J_{c_{R}-\frac{1}{2}}\left(\frac{m}{k^{\prime}}\right)-\frac{J_{c_{R}-\frac{1}{2}}\left(\frac{m}{k}\right)}{Y_{c_{R}-\frac{1}{2}}\left(\frac{m}{k}\right)} Y_{c_{R}-\frac{1}{2}}\left(\frac{m}{k^{\prime}}\right)\right) \\
& -\lambda^{2}\left(J_{c_{L}+\frac{1}{2}}\left(\frac{m}{k^{\prime}}\right)-\frac{J_{c_{L}-\frac{1}{2}}\left(\frac{m}{k}\right)}{Y_{c_{L}-\frac{1}{2}}\left(\frac{m}{k}\right)} Y_{c_{L}+\frac{1}{2}}\left(\frac{m}{k^{\prime}}\right)\right)\left(J_{c_{R}+\frac{1}{2}}\left(\frac{m}{k^{\prime}}\right)-\frac{J_{c_{R}-\frac{1}{2}}\left(\frac{m}{k}\right)}{Y_{c_{R}-\frac{1}{2}}\left(\frac{m}{k}\right)} Y_{c_{R}+\frac{1}{2}}\left(\frac{m}{k^{\prime}}\right)\right)=0 .
\end{aligned}
$$

This is the equation cited in the text as Eq. (23). The mass eigenvalues, $m$, are determined as solutions of this equation.

For the down-type quarks, we have to use $c_{L}=c_{q}, c_{R}=c_{\psi_{\bar{d}}}$ and $\lambda=y_{d} v_{H}$, instead of $c_{L}=c_{q}$, $c_{R}=c_{\psi_{\bar{u}}}$ and $\lambda=y_{u} v_{H}$. For the leptons, $c_{L}=c_{l}, c_{R}=c_{\psi_{\bar{e}}}$ and $\lambda=y_{e} v_{H}$. 


\section{References}

[1] S. Weinberg, Phys. Rev. D 13, 974 (1976); Phys. Rev. D 19, 1277 (1979); L. Susskind, Phys. Rev. D 20, 2619 (1979).

[2] D. B. Kaplan and H. Georgi, Phys. Lett. B 136, 183 (1984); D. B. Kaplan, H. Georgi and S. Dimopoulos, Phys. Lett. B 136, 187 (1984).

[3] J. M. Maldacena, Adv. Theor. Math. Phys. 2, 231 (1998) [Int. J. Theor. Phys. 38, 1113 (1999)] [arXiv:hep-th/9711200]; S. S. Gubser, I. R. Klebanov and A. M. Polyakov, Phys. Lett. B 428, 105 (1998) [arXiv:hep-th/9802109]; E. Witten, Adv. Theor. Math. Phys. 2, 253 (1998) [arXiv:hep-th/9802150].

[4] R. Contino, Y. Nomura and A. Pomarol, Nucl. Phys. B 671, 148 (2003) [arXiv:hepph/0306259]; T. Gherghetta and A. Pomarol, Phys. Rev. D 67, 085018 (2003) [arXiv:hep$\mathrm{ph} / 0302001]$.

[5] C. Csaki, C. Grojean, L. Pilo and J. Terning, arXiv:hep-ph/0308038.

[6] Y. Nomura, JHEP 0311, 050 (2003) [arXiv:hep-ph/0309189].

[7] C. Csaki, C. Grojean, H. Murayama, L. Pilo and J. Terning, arXiv:hep-ph/0305237.

[8] R. Barbieri, A. Pomarol and R. Rattazzi, arXiv:hep-ph/0310285.

[9] M. E. Peskin and T. Takeuchi, Phys. Rev. Lett. 65, 964 (1990); Phys. Rev. D 46, 381 (1992).

[10] L. Randall and R. Sundrum, Phys. Rev. Lett. 83, 3370 (1999) [arXiv:hep-ph/9905221].

[11] S. Dimopoulos and L. Susskind, Nucl. Phys. B 155, 237 (1979); E. Eichten and K. D. Lane, Phys. Lett. B 90, 125 (1980).

[12] B. Holdom, Phys. Rev. D 24, 1441 (1981); K. Yamawaki, M. Bando and K. i. Matumoto, Phys. Rev. Lett. 56, 1335 (1986); T. W. Appelquist, D. Karabali and L. C. R. Wijewardhana, Phys. Rev. Lett. 57, 957 (1986).

[13] G. 't Hooft, Nucl. Phys. B 72, 461 (1974).

[14] Y. Nomura, D. R. Smith and N. Weiner, Nucl. Phys. B 613, 147 (2001) [arXiv:hep$\mathrm{ph} / 0104041]$.

[15] W. D. Goldberger, Y. Nomura and D. R. Smith, Phys. Rev. D 67, 075021 (2003) [arXiv:hepph/0209158]; S. J. Huber and Q. Shafi, arXiv:hep-ph/0309252.

[16] N. Arkani-Hamed, A. G. Cohen and H. Georgi, Phys. Lett. B 516, 395 (2001) [arXiv:hepth/0103135]; T. Hirayama and K. Yoshioka, arXiv:hep-th/0311233. 
[17] T. Gherghetta and A. Pomarol, Nucl. Phys. B 586, 141 (2000) [arXiv:hep-ph/0003129]; S. J. Huber and Q. Shafi, Phys. Lett. B 498, 256 (2001) [arXiv:hep-ph/0010195].

[18] W. D. Goldberger and I. Z. Rothstein, Phys. Rev. Lett. 89, 131601 (2002) [arXiv:hepth/0204160]; arXiv:hep-th/0208060; Y. Nomura and D. R. Smith, Phys. Rev. D 68, 075003 (2003) [arXiv:hep-ph/0305214].

[19] A. Manohar and H. Georgi, Nucl. Phys. B 234, 189 (1984); H. Georgi and L. Randall, Nucl. Phys. B 276, 241 (1986); Z. Chacko, M. A. Luty and E. Ponton, JHEP 0007, 036 (2000) [arXiv:hep-ph/9909248]; Y. Nomura, Phys. Rev. D 65, 085036 (2002) [arXiv:hep$\mathrm{ph} / 0108170]$.

[20] N. Arkani-Hamed, M. Porrati and L. Randall, JHEP 0108, 017 (2001) [arXiv:hepth/0012148]; R. Rattazzi and A. Zaffaroni, JHEP 0104, 021 (2001) [arXiv:hep-th/0012248]; M. Perez-Victoria, JHEP 0105, 064 (2001) [arXiv:hep-th/0105048].

[21] See, for example, E. Witten, Nucl. Phys. B 160, 57 (1979).

[22] K. Agashe, A. Delgado, M. J. May and R. Sundrum, JHEP 0308, 050 (2003) [arXiv:hep$\mathrm{ph} / 0308036]$.

[23] H. Georgi, Nucl. Phys. B 363, 301 (1991); M. J. Dugan and L. Randall, Phys. Lett. B 264, 154 (1991); E. Gates and J. Terning, Phys. Rev. Lett. 67, 1840 (1991); M. A. Luty and R. Sundrum, Phys. Rev. Lett. 70 (1993) 529 [arXiv:hep-ph/9209255].

[24] B. Holdom, Phys. Lett. B 259, 329 (1991); P. Langacker and M. x. Luo, Phys. Rev. D 45, 278 (1992).

[25] C. Csaki, M. L. Graesser and G. D. Kribs, Phys. Rev. D 63, 065002 (2001) [arXiv:hepth/0008151]; J. F. Gunion, M. Toharia and J. D. Wells, arXiv:hep-ph/0311219.

[26] L. J. Hall, H. Murayama and Y. Nomura, Nucl. Phys. B 645, 85 (2002) [arXiv:hepth/0107245]; R. Sekhar Chivukula, D. A. Dicus and H. J. He, Phys. Lett. B 525, 175 (2002) [arXiv:hep-ph/0111016]; Y. Abe, N. Haba, Y. Higashide, K. Kobayashi and M. Matsunaga, Prog. Theor. Phys. 109, 831 (2003) [arXiv:hep-th/0302115].

[27] C. Csaki, C. Grojean, J. Hubisz, Y. Shirman and J. Terning, arXiv:hep-ph/0310355.

[28] M. Henningson and K. Sfetsos, Phys. Lett. B 431, 63 (1998) [arXiv:hep-th/9803251]; W. Muck and K. S. Viswanathan, Phys. Rev. D 58, 106006 (1998) [arXiv:hep-th/9805145]; J. Garriga and A. Pomarol, Phys. Lett. B 560, 91 (2003) [arXiv:hep-th/0212227].

[29] See, for example, T. Abe et al. [American Linear Collider Working Group Collaboration], in Proc. of the APS/DPF/DPB Summer Study on the Future of Particle Physics (Snowmass 2001) ed. N. Graf, arXiv:hep-ex/0106055; arXiv:hep-ex/0106057. 
[30] D. Atwood and G. Hiller, arXiv:hep-ph/0307251.

[31] K. Agashe, Talk given at the Second Workshop on the Discovery Potential of an Asymmetric B Factory at $10^{36}$ Luminosity, SLAC, Oct 2003.

[32] G. Burdman, arXiv:hep-ph/0310144.

[33] G. Burdman and I. Shipsey, arXiv:hep-ph/0310076.

[34] N. Arkani-Hamed, S. Dimopoulos and G. R. Dvali, Phys. Lett. B 429, 263 (1998) [arXiv:hepph/9803315]; I. Antoniadis, N. Arkani-Hamed, S. Dimopoulos and G. R. Dvali, Phys. Lett. B 436, 257 (1998) [arXiv:hep-ph/9804398].

[35] For example, R. Sundrum, Nucl. Phys. B 395, 60 (1993) [arXiv:hep-ph/9205203].

[36] G. F. Giudice, R. Rattazzi and J. D. Wells, Nucl. Phys. B 595, 250 (2001) [arXiv:hepph/0002178]; C. Csaki, M. L. Graesser and G. D. Kribs, in [25].

[37] H. Davoudiasl, J. L. Hewett, B. Lillie and T. G. Rizzo, arXiv:hep-ph/0312193. 